\title{
THE SPECTROSCOPICALLY DETERMINED SUBSTELLAR MASS FUNCTION OF THE ORION NEBULA CLUSTER
}

\author{
Catherine L. Slesnick, ${ }^{1}$ Lynne A. Hillenbrand, ${ }^{1}$ and John M. Carpenter ${ }^{1}$ \\ Department of of Astronomy, MS 105-24, California Institute of Technology, Pasadena, CA 91125; \\ cls@astro.caltech.edu, lah@astro.caltech.edu,jmc@astro.caltech.edu \\ Received 2004 January 28; accepted 2004 March 29
}

\begin{abstract}
We present a spectroscopic study of candidate brown dwarf members of the Orion Nebula cluster (ONC). We obtained new $J$ - and/or $K$-band spectra of $\sim 100$ objects within the ONC that are expected to be substellar on the basis of their $K$ magnitudes and $H-K$ colors. Spectral classification in the near-infrared of young low-mass objects is described, including the effects of surface gravity, veiling due to circumstellar material, and reddening. From our derived spectral types and existing near-infrared photometry, we construct an H-R diagram for the cluster. Masses are inferred for each object and used to derive the brown dwarf fraction and assess the mass function for the inner 5.'1 $\times 5$. $^{\prime} 1$ of the ONC, down to $\sim 0.02 M_{\odot}$. The logarithmic mass function rises to a peak at $\sim 0.2 M_{\odot}$, similar to previous initial mass function determinations derived from purely photometric methods but falls off more sharply at the hydrogen-burning limit before leveling through the substellar regime. We compare the mass function derived here for the inner $\mathrm{ONC}$ with those presented in recent literature for the sparsely populated Taurus cloud members and the rich cluster IC 348. We find good agreement between the shapes and peak values of the ONC and IC 348 mass distributions but little similarity between the ONC and Taurus results.
\end{abstract}

Subject headings: infrared: stars — open clusters and associations: individual (Orion Nebula cluster) stars: low-mass, brown dwarfs — stars: luminosity function, mass function stars: pre-main-sequence

\section{INTRODUCTION}

The stellar mass and age distributions in young star clusters can help answer some of the fundamental questions of cluster formation theory: Do all cluster members form in a single burst, or is star formation a lengthy process? Is the distribution of stellar masses formed during a single epoch within a cluster, also known as the initial mass function (IMF), universal, or does it vary with either star formation environment or time? While the stellar mass function has long been studied (e.g., Salpeter 1955), we are only recently beginning to explore the very low mass end of the distribution into the substellar regime. Identification of large unbiased samples of low-mass objects, especially in star-forming regions, is crucial to our understanding of the formation and early evolution of low-mass stars and brown dwarfs. Young stellar clusters are particularly valuable for examining the shape of the low-mass IMF because the lowest mass members have not yet been lost to dynamical evolution. Furthermore, contracting low-mass pre-main-sequence (PMS) stars and brown dwarfs are 2-3.5 orders of magnitude more luminous than their counterparts on the main sequence and thus can be more readily detected in large numbers. The dense molecular clouds associated with star-forming regions also reduce background field star contamination.

Because the Orion Nebula cluster (ONC) is one of the nearest massive star-forming regions to the Sun and the most populous young cluster within $\sim 2 \mathrm{kpc}$, it has been observed at virtually all wavelengths over the past several decades. However, only recently have increased sensitivities due to nearinfrared detectors on larger telescopes allowed us to begin to

\footnotetext{
${ }^{1}$ Visiting astronomer, W. M. Keck Observatory, which is operated as a scientific partnership among the California Institute of Technology, the University of California, and the National Aeronautics and Space Administration.
}

understand and characterize the extent of the ONC's young stellar and brown dwarf population, which, at $\lesssim 1-2 \mathrm{Myr}$, is just beginning to emerge from its giant molecular cloud.

Several recent studies have explored the ONC at substellar masses. Hillenbrand \& Carpenter (2000, hereafter HC00) present the results of an $H$ and $K$ imaging survey of the inner $5 ! 1 \times 5{ }^{\prime} 11$ region of the ONC. Observed magnitudes, colors, and star counts were used to constrain the shape of the ONC mass function across the hydrogen-burning limit down to $\sim 0.03 M_{\odot}$. They find evidence in the $\log$ - $\log$ mass function for a turnover above the hydrogen-burning limit, then a plateau into the substellar regime. A similar study by Muench et al. (2002, hereafter M02) uses $J, H$, and $K$ imaging of the ONC to derive an IMF that rises to a broad primary peak at the lowest stellar masses between $0.3 M_{\odot}$ and the hydrogen-burning limit before turning over and declining into the substellar regime. However, instead of a plateau through the lowest masses, M02 find evidence for a secondary peak between 0.03 and $0.02 M_{\odot}$. Luhman et al. (2000) use $H$ and $K$ infrared imaging and limited ground-based spectroscopy to constrain the mass function and again find a peak just above the substellar regime but then a steady decline through the lowest mass objects.

Generally speaking, $J, H$, and $K$ photometry alone is insufficient for deriving stellar/substellar masses, although it may be adequate in a statistical sense for estimating mass distributions given the right assumptions. The position of a young star in a near-infrared color-magnitude diagram (CMD) is dependent on mass, age, extinction, and the possible presence of a circumstellar disk. These characteristics affect the conversion of a star's infrared magnitude and color into its stellar mass. Unless the distributions of these parameters are known a priori, knowledge of the cluster's luminosity function alone is not sufficient to draw definitive conclusions about its 
mass function. In addition, cluster membership is often poorly known and statistical estimates concerning the extent and characterization of the field star population must be derived. In the case of the densely populated ONC, it has been suggested that the field star contamination is small but nonnegligible toward fainter magnitudes. HCO0 used a modified version of the Galactic star count model (Wainscoat et al. 1992) convolved with a local extinction map (derived from a $\mathrm{C}^{18} \mathrm{O}$ molecular line map) to estimate the field star contribution, which they found to constitute $\sim 5 \%$ of the stars down to their completeness limit at $K \sim 17.5$.

To study a cluster's IMF in more than just a statistical sense, spectroscopy is needed to confirm cluster membership of individual stars and uniquely determine location in the H-R diagram (and hence mass). We have obtained near-infrared spectra of 97 stars in the ONC. This wavelength regime (1$2.5 \mu \mathrm{m}$ ) is of extreme interest for very cool stars and brown dwarfs, not only because ultracool objects emit the bulk of their energy in the near-infrared, but also because this regime contains temperature-sensitive atomic, as well as broad molecular, features. In addition, there are several diagnostic lines that can be used as surface gravity indicators. From analysis of these data, combined with existing photometry and PMS evolutionary theory, we construct the cluster's IMF across the substellar boundary. We then compare our results with those found from previous studies, both of the ONC and of clusters similar in age to the ONC but that have different star-forming environments.

In $\S 2$ we describe our data acquisition and reduction. In $\S 3$ we present our spectra and methods for spectral classification. This section includes a discussion of the effects of extinction and veiling due to circumstellar disks. In $\S 4$ we create an H-R diagram for the stellar and substellar objects for which we have new spectral types. Section 5 contains our derivation of the ONC's IMF, and $\S 6$, our analysis and comparison with previous work.

\section{OBSERVATIONS}

\subsection{Infrared Spectroscopic Sample Selection}

Candidate substellar objects have been selected from the HC00 photometric survey for follow-up spectroscopy to determine whether their temperatures and surface gravities are consistent with those of brown dwarf objects at the age $(\sim 1 \mathrm{Myr})$ and distance $(480 \mathrm{pc})$ of the ONC. Figure 1 is a $\mathrm{CMD}$ showing the $\mathrm{HC} 00$ photometry. We transformed the isochrones and mass tracks of D'Antona \& Mazzitella $^{2}$ (1997, hereafter DM97; 1998) into the $K,(H-K)$ plane and selected stars for spectroscopy on the basis of their location in the CMD below a line that corresponds to the reddening vector originating at the hydrogen-burning limit $\left(M=0.08 M_{\odot}\right)$. In addition to stars selected from infrared photometry, several stars were followed up with infrared spectroscopy before the deep HCOO data became available on the basis of optical $(V$ and I) magnitudes and colors (Hillenbrand 1997, hereafter H97), indicating that they might be substellar.

We obtained new infrared spectra of 97 objects within the ONC; 81 selected from the HC00 work were observed using NIRSPEC ( $\S 2.2 .1$ ), and 16 selected from optical magnitudes and colors were observed using the infrared Cryogenic Spectrometer (CRSP; $\S 2.2 .2$ ). Our sample included $\sim 50 \%$ of

\footnotetext{
${ }^{2}$ The 1998 models are a web-only correction at less than $0.2 M_{\odot}$ to their original 1997 work.
}

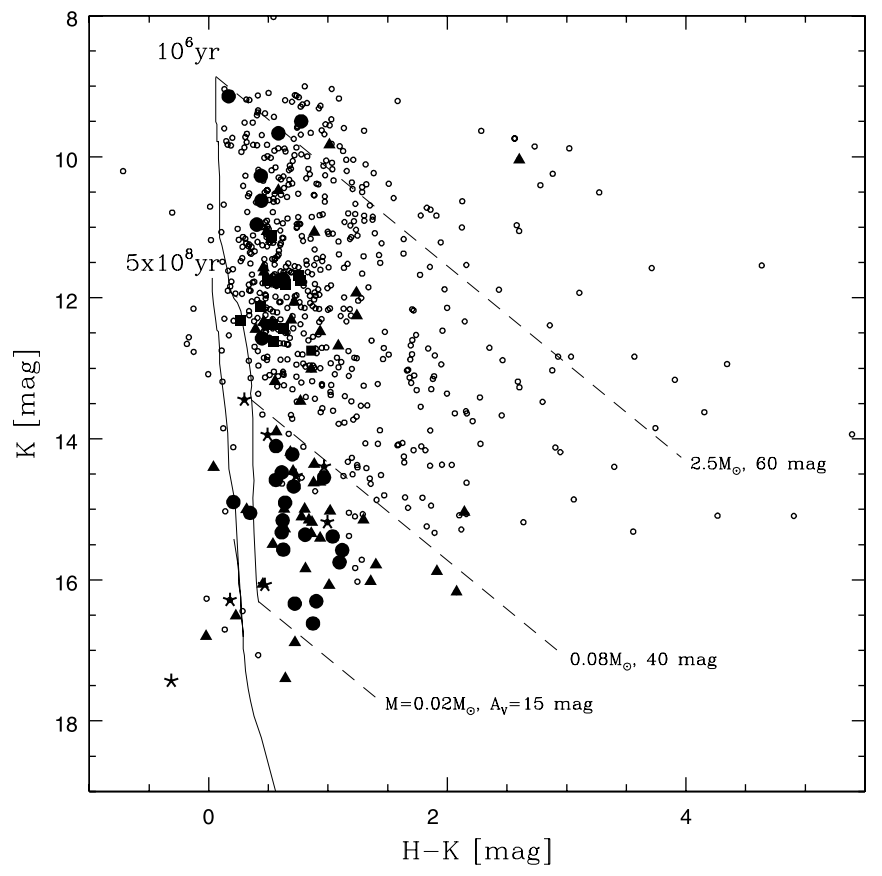

FIG. 1. $-K,(H-K)$ CMD for the ONC. Isochrones are from DM97 and have been transformed into the $K,(H-K)$ plane assuming the age ( $\$ 1 \mathrm{Myr})$ and distance $(480 \mathrm{pc})$ of the ONC. Dashed lines are reddening vectors emanating at the indicated masses from the $1 \mathrm{Myr}$ isochrone. Open circles represent $\mathrm{HC} 00$ photometry of the inner $5 !^{\prime} 1 \times 5{ }^{\prime} 1$ of the nebula. Sources for which we have $J$-band or $K$-band spectra taken with NIRSPEC are marked as filled circles and triangles, respectively. Stars were selected for spectroscopy on the basis of their location on the CMD below $0.08 M_{\odot}$. Often it was possible to place multiple stars on the slit because of the high stellar density of the cluster, allowing us to observe several brighter (more massive) stars. Stars indicate sources for which we have new red optical LRIS spectral classifications. Filled squares represent sources (mostly in the outer nebula) for which we have $J$ - and $K$-band spectra taken with the CRSP.

the stars in the $\mathrm{HC} 00$ survey area expected to be brown dwarfs on the basis of their $K$ and $H-K$ magnitudes and colors, down to the completeness limit of $K \sim 17.5$. Often it was possible to place multiple stars on the slit because of the high stellar density of the cluster, allowing us to observe several brighter stars, some with known spectral types that could be used as secondary spectral standards.

\subsection{Infrared Data Acquisition}

\subsubsection{NIRSPEC Data}

Near-infrared spectra of candidates selected from infrared photometry were taken with the NIRSPEC spectrograph on the Keck II Telescope on Mauna Kea, Hawaii. This nearinfrared spectrograph has a $1024 \times 1024 \mathrm{InSb}$ array with $27 \mu \mathrm{m}$ pixels. We used the low-resolution mode of the camera with an entrance slit of $42^{\prime \prime} \times 00^{\prime \prime} 72$, resulting in a resolving power of $R \sim 1400$. Stars were placed in the entrance slit using a $K$-band guide camera. $J$-band spectra were taken on 2002 February 2 and 3 through the NIRSPEC-3 filter, which spans the wavelength range $1.143-1.375 \mu \mathrm{m}$. Typical exposure times were $\sim 300$ s. $K$-band data were taken on 2002 November 29 and December 1 and 2 through the $K^{\prime}$ filter $(1.950-2.295 \mu \mathrm{m})$. This filter offers less wavelength coverage than either the $K$ or the NIRSPEC-7 filters; however, it afforded us extremely high transmission, particularly at the short-wavelength end. The $K^{\prime}$ filter has more than $90 \%$ transmission from 2 to $2.95 \mu \mathrm{m}$, whereas the NIRSPEC-7 filter gives an average of $\sim 80 \%$ transmission (sometimes as 
low as $70 \%$ ) in this range, and the $K$ filter gives an average of $70 \%$. Our aim when taking the $K$-band spectra was to observe as many faint objects as possible so that we would have a statistically large enough sample of known cluster members with spectral types (and therefore, masses) from which to construct an accurate IMF. We felt it would be a more effective use of limited telescope time to use the $K^{\prime}$ filter so that we could observe more and fainter objects with shorter exposure times. We were still able to make use of two of the three molecular absorption features between 2 and $2.5 \mu \mathrm{m}$ commonly utilized for classification of low-mass stars (see $\S 3.1$ ). Typical exposure times for the $K$-band data were $300-600 \mathrm{~s}$.

For each object, we took exposures in sets of three, nodding the star along the slit between each exposure. For our fainter targets, two or three sets of observations were required. In most cases we were able to observe multiple objects in a single exposure by rotating the slit position angle. Telluric reference stars were observed throughout the night over a wide range in air mass. We observed $\mathrm{O}$ and $\mathrm{G}$ stars whose spectra have relatively few absorption features in the $J$ and $K$ bands at our resolution and may be used as telluric templates. $\mathrm{NeAr}$ arc lamp spectra and internal flats used in rectifying the images ( $\operatorname{see} \S 2.3$ ) were taken at the start and end of each night.

\subsubsection{CRSP Data}

For sources selected from optical $I$ and $V-I$ magnitudes and colors, spectra were obtained with CRSP on the Mayall $4 \mathrm{~m}$ telescope at Kitt Peak National Observatory on the nights of 1998 January 16-19. The instrument is a cooled grating spectrograph with a $256 \times 256 \mathrm{InSb}$ detector. Low-resolution $(R=1000)$ spectra were collected using a 200 line $\mathrm{mm}^{-1}$ grating in the $Y, J, H$, and $K$ bands (third, second, second, and first orders, respectively). The slit subtended 1".0, with the infrared seeing typically 1 ."9-1".2. Stars were positioned in the slit via a visible guide camera, and data were collected in a standard two-beam beam-switching mode. Integration times ranged from 0.316 (minimum readout time) to $120 \mathrm{~s}$ for individual frames. A0 and G2 telluric standard stars were observed for correction of spectral standards, while the $\mathrm{O} 7 \mathrm{star}$ $\theta^{1} \mathrm{C}$ Ori was used for program stars. Wavelength calibration was established from exposures of a HeNeAr arc lamp, while dome flats were collected with and without incandescent illumination of a white screen mounted inside the telescope dome.

\subsection{Infrared Data Reduction}

\subsubsection{NIRSPEC Data}

We reduced the NIRSPEC data within IRAF, applying both standard tasks and custom techniques developed for reducing NIRSPEC data. All sources, including standards, were preprocessed and extracted in the same manner as outlined below.

Bad-pixel masks were created by median-combining dark exposures taken at the beginning of each night. The mask was then applied to all images within a given night using the IRAF flvmask task, which replaces bad pixels by interpolating along the spectral direction. Cosmic rays were removed using qzap, written by Mark Dickenson. Since raw NIRSPEC data are tilted in both the spatial and dispersion directions, we used custom IRAF software written by Gregory D. Wirth (available publicly on the NIRSPEC Web site) to rectify the data. This software first removes shifts in the $x$ - (spatial) direction using a spatial map, created from a flat-field exposure, to align the edge of an image along a single column. Next, arc lamp spectra are used to align emission features along image rows in the wavelength direction by applying a second-order Chebyshev fit.

We did not flat-field the data because we found that the internal flats did not uniformly illuminate the detector in the lower resolution mode. In addition, for the $J$-band data the presence of ice on the Dewar window caused time-variable streaks on the flat-field images in the spectral direction. While nonuniform illumination of the flat-field images does not cause a large problem for observations concerned only with narrow absorption or emission lines, we are interested in the strength of broad molecular absorption bands measured as relative flux levels at different parts of the spectrum. Therefore, it is essential that the data be flat in the dispersion direction. We found that using the flat-field images on the standard-star observations introduced error rather than corrected it. Instead, division by a telluric reference star corrected adequately for any nonuniform features in the dispersion direction of the detector. Once the effectiveness of this method was confirmed with our sequences of standard stars, we applied it to our ONC spectra (see below).

To remove background night-sky and nebular emission lines, we used one of the adjacent nod position images to create a sky frame (or an average of two nod position images). Simple image subtraction does not work for these data because the background emission varies on short timescales. Instead, we used the median value along each row to create background emission frames free from stellar continuum for both the target and sky frames. The sky emission frame was then scaled at each row to match the relative intensities of the emission lines on the target emission frame. This new sky frame was then subtracted from the original target frame. Because our objects are located in the Orion Nebula, whose spectrum contains very strong emission features that can vary on extremely small spatial scales (a few arcseconds), some residual emission/absorption areas were sometimes left after sky subtraction. To correct for these, individual background regions were determined and subtracted from the source during spectral extraction (using apall within IRAF).

Each spectrum was wavelength-calibrated using sky emission lines, except the short exposures for which the next closest observation in time was used. A telluric spectrum was produced for each extraction from a weighted average of the two flux standards closest in air mass, thereby creating a standard spectrum at the air mass of the object. For most objects we used $\theta^{1} \mathrm{C}$ Ori as the standard star both because of its nearly featureless spectrum and because its location near the center of the Orion Nebula makes it easy to observe at representative air masses. The only exceptions were some of the M-type standard stars, particularly those in Upper Sco, which were observed at air masses $\geq 1$.6. For these objects we used HR 4498 (G0 V) as a telluric standard after interpolating over the few detectable absorption features in its spectrum. Finally, we averaged together all spectra for a given object and multiplied by a synthetic blackbody spectrum appropriate for the temperature of the telluric standard used. The spectra were normalized to have an average flux of unity (over the entire wavelength region within the filter) for analysis.

Figure 2 shows two NIRSPEC spectra representative of the typical signal-to-noise ratio $(\mathrm{S} / \mathrm{N})$ for our program objects. We have chosen to show these objects because they were observed during both observing runs (along with HC 383, HC 509, and several of the standards) as a consistency check. 

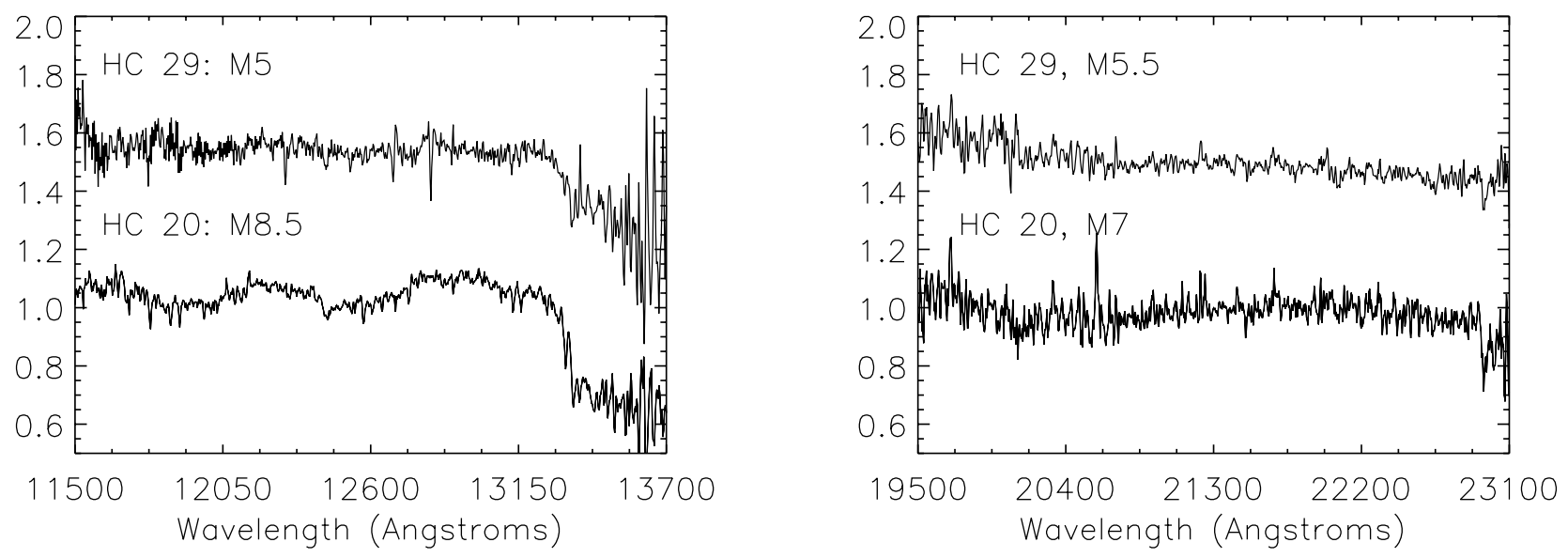

FIG. 2.-NIRSPEC spectra representative of the typical S/N for our program objects. Both targets have relatively low extinction $\left(A_{V}<5\right)$. Derived spectral types are indicated and demonstrate the degree of agreement between $J$ - and $K$-band spectral types when both data are available.

In addition, they have relatively low extinction $\left(A_{V}<5\right)$ and therefore can be more easily compared with previously published spectra of objects at the same temperatures (see $\S 3.4$ ). The few residual emission lines from the background nebula have been interpolated over. In general, we find agreement to within errors between $J$ - and $K$-band spectral types when both data are available. A detailed description of our spectral classification methods, including a discussion of reddening estimates, is given in $\S 3$. Typical signal-to-noise ratios of the ONC spectra were $\sim 10-70$ pixel $^{-1}$, with nearly all spectra classifiable.

\subsubsection{CRSP Data}

Standard image processing was accomplished using IRAF. After linearization, image trimming, and bad-pixel correction, individual beam-switched pairs of spectra were subtracted to effect a first-order sky subtraction and to remove dark current and bias offsets. Normalized flat fields were constructed from median-filtered combinations of "lights-on" minus "lightsoff" dome flats and used to flat-field the data frames. Spectra were extracted using the apall task and combined using a fluxweighted average. The dispersion solution was derived from extracted arc spectra and individually adjusted using crosscorrelation techniques to bring the spectra to a common wavelength zero point. This step was necessary because of the continual shifts of the grating required to obtain data in all four near-infrared atmospheric windows. To remove atmospheric and instrumentation effects from the data, the combined spectra were divided by telluric standards over which Paschen and Brackett series lines were first interpolated. To preserve the intrinsic spectral energy distributions of the program stars the telluric-divided spectra were multiplied by a blackbody function appropriate to the adopted telluric standard. The final spectra were normalized to have an average flux of unity over the entire spectral range. For the analysis presented here we use only the $J$-band and $K$-band spectra from this data set.

\subsection{Optical Spectroscopy}

We have compared the spectral types derived from our new infrared spectra with those obtained from optical spectra, either as presented in the tables of $\mathrm{H} 97$ or as newly updated by us on the basis of data obtained with the Keck II LRIS. Data were obtained with LRIS on six different occasions between 1998 January and 2002 January by us and by additional observers including N. Reid and B. Schaefer. Both single-slit and multislit modes were employed. Stars for the single-slit observations were selected in a manner similar to that employed for the CRSP observations described above, namely, on the basis of location in the $I,(V-I) \mathrm{CMD}$. The multislit observations were focused similarly to the NIRSPEC observations described above, primarily on the brighter substellar candidates from the $K$ and $H-K$ survey of $\mathrm{HCO0}$. Objects for which we have classifiable LRIS spectra are shown as stars points on Figure 1.

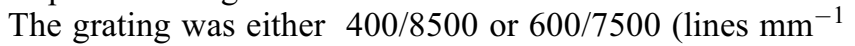
blaze) depending on the run, producing $R \sim 1400$ spectra nominally over $6000-10500 \AA$, with shifts in spectral coverage of up to $\pm 1000 \AA$ for the multislit data. Single-slit observations were typically $240-600 \mathrm{~s}$ integrations, whereas the multislit data were taken in stacks of five to eight $600 \mathrm{~s}$ integrations to avoid nebular saturation perpendicular to the dispersion direction and thus across the slitlets. Data were reduced, after median filtering and stacking the multislit data, using standard techniques of bias-subtraction, flat-fielding, and spectral extraction, with particular attention needed because of the strong and spatially variable nebular emission. Spectra were classified using a depth-of-feature analysis as described in $\mathrm{H} 97$, with the TiO and VO bands the most important spectral diagnostics in the M-type range of interest in this paper.

Optical spectral types are presented in Tables 1 and 2 for comparison with our infrared spectral types. Types that are not footnoted come from the new LRIS spectra, whereas footnoted types are from $\mathrm{H} 97$.

\section{INFRARED SPECTRAL CLASSIFICATION}

To ensure that we could accurately classify our program spectra we took a range of spectral main-sequence standards using NIRSPEC: M0-M9 at $J$ band and $\mathrm{K} 7-\mathrm{L} 3$ at $K$ band. We also took spectra of mid- to late $\mathrm{M}$ lower surface gravity stars in Upper Sco and Praesepe, as well as field giants. For the $J$-band data we were able to include electronically available standard spectra of nearby field dwarfs taken by the Leggett group $^{3}$ because our classification indices rely on the depth of $\mathrm{H}_{2} \mathrm{O}$ and $\mathrm{FeH}$ absorption features rather than the shape of the continuum (the continuum shape was later considered in comparison with our NIRSPEC standards when possible; see

\footnotetext{
${ }^{3}$ See www.jach.hawaii.edu/ skl/publications.html.
} 
TABLE 1

Band Indices and Spectral Types for the Inner 5.' $1 \times 5$.' 1 of the ONC

\begin{tabular}{|c|c|c|c|c|c|c|c|c|c|}
\hline \multirow[b]{2}{*}{$\mathrm{ID}^{\mathrm{a}}$} & \multirow[b]{2}{*}{$K^{\mathrm{a}}$} & \multicolumn{2}{|c|}{$J$ BAND } & \multicolumn{2}{|c|}{$K$ BAND } & \multicolumn{2}{|c|}{ SPECTRAL Type } & \multirow[b]{2}{*}{ Gravity $^{\mathrm{d}}$} & \multirow[b]{2}{*}{ COMMENTs $^{\mathrm{e}}$} \\
\hline & & $\mathrm{H}_{2} \mathrm{O}-1$ & $\mathrm{FeH}$ & $\mathrm{H}_{2} \mathrm{O}-2$ & $\mathrm{H}_{2}$ & Optical $^{\mathrm{b}}$ & $\mathrm{IR}^{\mathrm{c}}$ & & \\
\hline 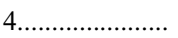 & 14.91 & 0.799 & 1.017 & $\ldots$ & $\ldots$ & M4.5 & M5.5 & Low & \\
\hline $15 \ldots \ldots \ldots \ldots \ldots \ldots$ & 15.36 & 0.921 & 0.974 & $\ldots$ & $\ldots$ & M0-M1 & M3.5 & $\ldots$ & \\
\hline 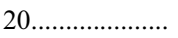 & 14.19 & 0.639 & 0.959 & 0.985 & -0.018 & $\ldots$ & M8 & Low & \\
\hline $22 \ldots \ldots \ldots \ldots \ldots \ldots$ & 13.19 & $\ldots$ & $\ldots$ & 0.997 & -0.014 & $\ldots$ & M8 & Int & Possible NIR emission lines \\
\hline $25 \ldots \ldots \ldots \ldots \ldots \ldots \ldots$ & 13.90 & $\ldots$ & $\ldots$ & 1.189 & -0.167 & $M:{ }^{f}$ & M1 & Low/int & \\
\hline $27 \ldots \ldots \ldots \ldots \ldots \ldots$ & 16.30 & 0.801 & 0.962 & $\ldots$ & $\ldots$ & $\ldots$ & M5 & Low & \\
\hline $29 \ldots \ldots \ldots \ldots \ldots \ldots$ & 11.74 & 0.827 & 0.987 & 1.032 & -0.040 & $\ldots$ & M5 & Low & \\
\hline $30 \ldots \ldots \ldots \ldots \ldots \ldots$ & 15.05 & 0.877 & 1.020 & $\ldots$ & $\ldots$ & $\mathrm{M} 0-3$ & M2 & Low & Ca IIe, possible NIR emission lines \\
\hline $35 \ldots \ldots \ldots \ldots \ldots \ldots \ldots$ & 11.11 & $\ldots$ & $\ldots$ & $\ldots$ & $\ldots$ & $\mathrm{M} 3.5^{\mathrm{f}}$ & M7: ${ }^{g}$ & $\ldots$ & \\
\hline 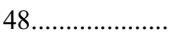 & 15.57 & 0.839 & 0.974 & $\ldots$ & $\ldots$ & $\ldots$ & M4 & Low & \\
\hline $51 \ldots \ldots \ldots \ldots \ldots \ldots$ & 11.63 & $\ldots$ & $\ldots$ & 1.017 & -0.013 & $\ldots$ & M5 & Int & \\
\hline 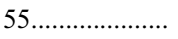 & 14.10 & 0.736 & 0.934 & $\ldots$ & $\ldots$ & $\ldots$ & M8 & Low & Possible NIR emission lines \\
\hline $59 \ldots \ldots \ldots \ldots \ldots \ldots \ldots$ & 16.29 & $\ldots$ & $\ldots$ & $\ldots$ & $\ldots$ & K8-M3 & $\ldots$ & $\ldots$ & \\
\hline $62 \ldots \ldots \ldots \ldots \ldots \ldots$ & 15.34 & $\ldots$ & $\ldots$ & 0.879 & 0.039 & $\ldots$ & M9 & Low & \\
\hline 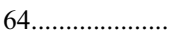 & 14.52 & $\ldots$ & $\ldots$ & $\ldots$ & $\ldots$ & M7-9 & $\ldots$ & Low & \\
\hline $70 \ldots \ldots \ldots \ldots \ldots \ldots \ldots$ & 15.75 & 0.624 & 0.870 & $\ldots$ & $\ldots$ & $\ldots$ & M9 & Low & \\
\hline 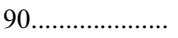 & 13.01 & $\ldots$ & $\ldots$ & 0.953 & 0.030 & $\ldots$ & M7.5 & Int & \\
\hline 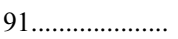 & 14.90 & 0.892 & 1.093 & $\ldots$ & $\ldots$ & $\ldots$ & $<\mathrm{K} 7^{\mathrm{h}}$ & $\ldots$ & Possible NIR emission lines \\
\hline 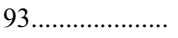 & 9.50 & 0.859 & 0.982 & $\ldots$ & $\ldots$ & $M 3 e^{f}$ & M3 & High & Possible NIR emission lines \\
\hline $111 \ldots \ldots \ldots \ldots \ldots \ldots$ & 15.38 & 0.704 & 0.943 & $\ldots$ & $\ldots$ & $\ldots$ & M9 & Low & \\
\hline $114 \ldots \ldots \ldots \ldots \ldots \ldots$ & 13.94 & $\ldots$ & $\ldots$ & $\ldots$ & $\ldots$ & M7 & $\ldots$ & Low & \\
\hline 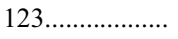 & 14.61 & $\ldots$ & $\ldots$ & 0.977 & 0.031 & M0-5 & M7.5 & Low & \\
\hline $127 \ldots \ldots \ldots \ldots \ldots$ & 14.55 & 0.960 & 1.021 & $\ldots$ & $\ldots$ & $\ldots$ & M0 & Int & \\
\hline $143 \ldots \ldots \ldots \ldots \ldots$ & 15.00 & $\ldots$ & $\ldots$ & 1.152 & -0.377 & $<\mathrm{M} 2:$ & $\mathrm{M} 2.5$ & Low & \\
\hline $162 \ldots \ldots \ldots \ldots \ldots \ldots$ & 12.48 & $\ldots$ & $\ldots$ & 0.979 & -0.035 & $\ldots$ & M5.5 & Low & \\
\hline 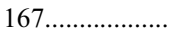 & 15.02 & $\ldots$ & $\ldots$ & 0.942 & -0.007 & M6-8 & M7.5 & Low & \\
\hline 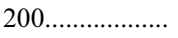 & 15.49 & $\ldots$ & $\ldots$ & 1.145 & -0.159 & $\ldots$ & M3 & $\ldots$ & \\
\hline 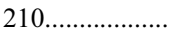 & 14.68 & 0.732 & 0.996 & $\ldots$ & $\ldots$ & >M6: & M7 & Low & $\mathrm{Ca}$ IIе \\
\hline $212 \ldots \ldots \ldots \ldots \ldots$ & 14.22 & 0.683 & 0.904 & $\ldots$ & $\ldots$ & $\ldots$ & M9 & Low & \\
\hline $221 \ldots \ldots \ldots \ldots \ldots . . . . . . . .$. & 14.46 & $\ldots$ & $\ldots$ & 0.949 & -0.038 & $\ldots$ & M7.5 & Low & \\
\hline $227 \ldots \ldots \ldots \ldots \ldots \ldots$ & 10.96 & 0.892 & 1.105 & $\ldots$ & $\ldots$ & M1 & $<\mathrm{K} 7^{\mathrm{h}}$ & $\ldots$ & \\
\hline $237 \ldots \ldots \ldots \ldots \ldots \ldots$ & 13.44 & $\ldots$ & $\ldots$ & $\ldots$ & $\ldots$ & M2 & $\ldots$ & Low & Ca IIе \\
\hline 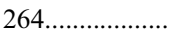 & 12.41 & $\ldots$ & $\ldots$ & $\ldots$ & $\ldots$ & $M 6^{\mathrm{f}}$ & $\mathrm{M}^{\mathrm{g}}$ & $\ldots$ & \\
\hline 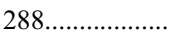 & 10.62 & 0.921 & 0.982 & $\ldots$ & $\ldots$ & $K 8^{\mathrm{f}}$ & M1 & High & Possible NIR emission lines \\
\hline 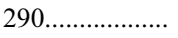 & 13.47 & $\ldots$ & $\ldots$ & 0.989 & -0.013 & $\ldots$ & M5.5: & $\ldots$ & \\
\hline 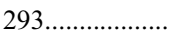 & 9.14 & 0.700 & 1.283 & $\ldots$ & $\ldots$ & B8 & $<\mathrm{K} 7^{\mathrm{h}}$ & Low & \\
\hline $316 \ldots \ldots \ldots \ldots \ldots \ldots$ & 15.18 & $\ldots$ & $\ldots$ & $\ldots$ & $\ldots$ & M3.5-5 & $\ldots$ & Low & $\mathrm{Ca}$ пाе \\
\hline 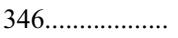 & 10.48 & $\ldots$ & $\ldots$ & 1.120 & -0.106 & $\ldots$ & M2 & Low/int & \\
\hline $355 \ldots \ldots \ldots \ldots \ldots \ldots$ & 12.35 & $\ldots$ & $\ldots$ & 1.054 & -0.067 & $\ldots$ & M5 & Int & Possible NIR emission lines \\
\hline $365 \ldots \ldots \ldots \ldots \ldots . . . . . . . .$. & 15.40 & $\ldots$ & $\ldots$ & 0.918 & 0.004 & $\ldots$ & M7: & $\ldots$ & \\
\hline 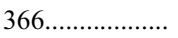 & 12.68 & $\ldots$ & $\ldots$ & 0.926 & 0.024 & $\ldots$ & M7.5 & Low & Possible NIR emission lines \\
\hline 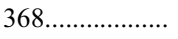 & 11.07 & $\ldots$ & $\ldots$ & 1.014 & -0.025 & $\ldots$ & M5 & Int & Possible $\mathrm{H}_{2}$ and He I P Cygni profile \\
\hline $372 \ldots \ldots \ldots \ldots \ldots \ldots$ & 14.48 & 0.677 & 0.958 & $\ldots$ & $\ldots$ & $\ldots$ & M9 & Low & \\
\hline $381 \ldots \ldots \ldots \ldots \ldots \ldots$ & 11.06 & $\ldots$ & $\ldots$ & 1.129 & -0.112 & $M 2^{f}$ & M1.5-4 & Int & \\
\hline $383 \ldots \ldots \ldots \ldots \ldots$ & 14.36 & 0.922 & 0.869 & 1.066 & -0.121 & $\ldots$ & M4 & Low & Possible NIR emission lines \\
\hline 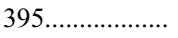 & 9.83 & $\ldots$ & $\ldots$ & 1.016 & -0.040 & $\ldots$ & M2: & $\ldots$ & \\
\hline $396 \ldots \ldots \ldots \ldots \ldots \ldots$ & 11.93 & $\ldots$ & $\ldots$ & 1.006 & -0.026 & $M:^{f}$ & M4-8 & Int & Possible NIR emission lines \\
\hline 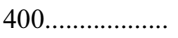 & 15.16 & 0.694 & 0.913 & $\ldots$ & $\ldots$ & M7-8 & M9 & Low & \\
\hline $403 \ldots \ldots \ldots \ldots \ldots \ldots$ & 15.15 & $\ldots$ & $\ldots$ & 0.930 & 0.005 & $\ldots$ & M7 & Low & Possible NIR emission lines \\
\hline 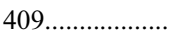 & 14.39 & $\ldots$ & $\ldots$ & $\ldots$ & $\ldots$ & M0 & $\ldots$ & $\ldots$ & \\
\hline $429 \ldots \ldots \ldots \ldots \ldots \ldots$ & 15.11 & $\ldots$ & $\ldots$ & 0.938 & 0.031 & M7-9 & M7.5 & Low & \\
\hline 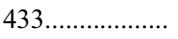 & 15.58 & 0.695 & 0.994 & $\ldots$ & $\ldots$ & $\ldots$ & M8 & Low & \\
\hline $434 \ldots \ldots \ldots \ldots \ldots \ldots$ & 10.04 & $\ldots$ & $\ldots$ & 0.949 & 0.022 & $\ldots$ & M2 & $\ldots$ & Possible He I P Cygni profile \\
\hline $454 \ldots \ldots \ldots \ldots \ldots \ldots$ & 12.06 & $\ldots$ & $\ldots$ & 1.055 & -0.068 & $\ldots$ & M2 & High & \\
\hline $455 \ldots \ldots \ldots \ldots \ldots . . . . . . . . .$. & 15.84 & $\ldots$ & $\ldots$ & 0.995 & -0.066 & $\ldots$ & M2-6 & $\ldots$ & \\
\hline $459 \ldots \ldots \ldots \ldots \ldots \ldots$ & 12.34 & $\ldots$ & $\ldots$ & 1.072 & -0.051 & $\ldots$ & M6 & Int & \\
\hline 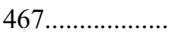 & 11.17 & $\ldots$ & $\ldots$ & 1.056 & -0.050 & $\mathrm{M} 2-4^{\mathrm{f}}$ & M6 & High & \\
\hline $469 \ldots \ldots \ldots \ldots \ldots \ldots$ & 14.74 & $\ldots$ & $\ldots$ & $\ldots$ & $\ldots$ & M & $\ldots$ & $\ldots$ & \\
\hline $509 \ldots \ldots \ldots \ldots \ldots$ & 15.33 & 0.900 & 0.988 & 0.986 & -0.126 & $\mathrm{M} 2-5$ & M2-7 & $\ldots$ & \\
\hline $515 \ldots \ldots \ldots \ldots \ldots \ldots$ & 15.88 & $\ldots$ & $\ldots$ & 0.804 & 0.088 & $\ldots$ & M7: & Low & \\
\hline $529 \ldots \ldots \ldots \ldots \ldots \ldots$ & 16.17 & $\ldots$ & $\ldots$ & 0.775 & 0.151 & $\ldots$ & M8 & Low & \\
\hline $543 \ldots \ldots \ldots \ldots \ldots \ldots$ & 10.27 & 0.931 & 0.977 & $\ldots$ & $\ldots$ & $\mathrm{M} 2.5^{\mathrm{f}}$ & M1 & Int/high & Possible NIR emission lines \\
\hline $553 \ldots \ldots \ldots \ldots \ldots \ldots$ & 12.31 & $\ldots$ & $\ldots$ & 1.043 & -0.035 & $\ldots$ & M5 & Low/int & Possible NIR emission lines \\
\hline $555 \ldots \ldots \ldots \ldots \ldots \ldots$ & 11.58 & $\ldots$ & $\ldots$ & 1.090 & -0.049 & $\ldots$ & M6 & $\ldots$ & \\
\hline
\end{tabular}


TABLE 1 - Continued

\begin{tabular}{|c|c|c|c|c|c|c|c|c|c|}
\hline \multirow[b]{2}{*}{$\mathrm{ID}^{\mathrm{a}}$} & \multirow[b]{2}{*}{$K^{\mathrm{a}}$} & \multicolumn{2}{|c|}{$J$ BAND } & \multicolumn{2}{|c|}{$K$ BAND } & \multicolumn{2}{|c|}{ SPECTRAL TyPe } & \multirow[b]{2}{*}{ GRAVItY $^{\mathrm{d}}$} & \multirow[b]{2}{*}{ Comments $^{\mathrm{e}}$} \\
\hline & & $\mathrm{H}_{2} \mathrm{O}-1$ & $\mathrm{FeH}$ & $\mathrm{H}_{2} \mathrm{O}-2$ & $\mathrm{H}_{2}$ & Optical $^{b}$ & $\mathrm{IR}^{\mathrm{c}}$ & & \\
\hline $559 \ldots \ldots \ldots \ldots \ldots . . . . . . . .$. & 14.40 & $\ldots$ & $\ldots$ & 0.958 & -0.017 & $\ldots$ & M8 & Low & Possible NIR emission lines \\
\hline $565 \ldots \ldots \ldots \ldots \ldots$ & 15.00 & $\ldots$ & $\ldots$ & 0.930 & 0.048 & $\ldots$ & M8 & Low & \\
\hline $568 \ldots \ldots \ldots \ldots \ldots . .$. & 15.00 & $\ldots$ & $\ldots$ & 0.808 & 0.172 & $\ldots$ & $<\mathrm{K} 7^{\mathrm{h}}$ : & $\ldots$ & \\
\hline $594 \ldots \ldots \ldots \ldots \ldots \ldots$ & 14.58 & 0.776 & 1.039 & $\ldots$ & $\ldots$ & $\ldots$ & M7.5 & Low & \\
\hline $600 \ldots \ldots \ldots \ldots \ldots \ldots$ & 12.25 & $\ldots$ & $\ldots$ & 0.979 & 0.056 & $\ldots$ & M5 & Low & \\
\hline $684 \ldots \ldots \ldots \ldots \ldots . . . . . . . .$. & 14.66 & $\ldots$ & $\ldots$ & 1.094 & -0.081 & $\ldots$ & $\mathrm{M} 0-4$ & $\ldots$ & \\
\hline 687.................... & 15.28 & $\ldots$ & $\ldots$ & 1.110 & -0.118 & $\ldots$ & M0-4 & $\ldots$ & \\
\hline 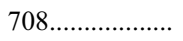 & 15.04 & $\ldots$ & $\ldots$ & 0.905 & 0.098 & $\ldots$ & M4 & Low & \\
\hline 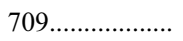 & 16.06 & $\ldots$ & $\ldots$ & 0.854 & 0.041 & $\ldots$ & M5 & Low & \\
\hline $721 \ldots \ldots \ldots \ldots \ldots \ldots$ & 15.18 & $\ldots$ & $\ldots$ & 0.995 & -0.102 & $\ldots$ & M3.5: & $\ldots$ & \\
\hline $722 \ldots \ldots \ldots \ldots \ldots \ldots$ & 17.43 & $\ldots$ & $\ldots$ & $\ldots$ & $\ldots$ & L0 & $\ldots$ & High & \\
\hline $723 \ldots \ldots \ldots \ldots \ldots \ldots$ & 16.07 & $\ldots$ & $\ldots$ & $\ldots$ & $\ldots$ & M3 & $\ldots$ & Low & \\
\hline $724 \ldots \ldots \ldots \ldots \ldots \ldots$ & 16.51 & $\ldots$ & $\ldots$ & 1.218 & -0.044 & $\ldots$ & M6: & $\ldots$ & \\
\hline $725 \ldots \ldots \ldots \ldots \ldots \ldots$ & 16.07 & $\ldots$ & $\ldots$ & 0.916 & 0.041 & $\ldots$ & M7: & $\ldots$ & \\
\hline $728 \ldots \ldots \ldots \ldots \ldots \ldots$ & 16.34 & 0.772 & 1.074 & $\ldots$ & $\ldots$ & $\ldots$ & M5.5 & Low & \\
\hline $729 \ldots \ldots \ldots \ldots \ldots$ & 16.02 & $\ldots$ & $\ldots$ & 0.861 & 0.032 & $\ldots$ & M7 & Low & Possible NIR emission lines \\
\hline $730 \ldots \ldots \ldots \ldots \ldots . . .$. & 15.15 & $\ldots$ & $\ldots$ & 1.111 & -0.114 & $\ldots$ & M4 & Low & Possible NIR emission lines \\
\hline $731 \ldots \ldots \ldots \ldots \ldots \ldots$ & 15.79 & $\ldots$ & $\ldots$ & 1.061 & -0.125 & $\ldots$ & K7: & $\ldots$ & \\
\hline $732 \ldots \ldots \ldots \ldots \ldots \ldots$ & 16.80 & $\ldots$ & $\ldots$ & 1.029 & 0.046 & $\ldots$ & M2.5: & $\ldots$ & \\
\hline $743 \ldots \ldots \ldots \ldots \ldots \ldots$ & 16.89 & $\ldots$ & $\ldots$ & 0.997 & -0.055 & $\ldots$ & M6: & $\ldots$ & \\
\hline $749 \ldots \ldots \ldots \ldots \ldots$ & 17.40 & $\ldots$ & $\ldots$ & 1.001 & 0.075 & $\ldots$ & M8 & $\ldots$ & \\
\hline 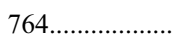 & 14.62 & $\ldots$ & $\ldots$ & 0.974 & -0.194 & $\mathrm{~K} 8-\mathrm{M} 3$ & M7.5: & $\ldots$ & \\
\hline
\end{tabular}

${ }^{\text {a }}$ IDs and $K$ magnitudes from HC00.

${ }^{\mathrm{b}}$ Optical spectral types are from LRIS data presented here unless otherwise noted.

${ }^{\mathrm{c}}$ Uncertainties in infrared spectral types are \pm 1.5 subclasses unless otherwise noted. A colon indicates that the spectrum had lower $\mathrm{S} / \mathrm{N}$, and the classification is less certain.

"For $J$-band spectra, an object was given a gravity classification of "low" if it had no detectable atomic absorption lines and "high" if it had strong lines, similar to those seen in spectra of dwarf standards of same spectral types. A classification of "int" indicates that absorption lines were present but not as strong as those in dwarf stars at the same temperature. For $K$-band spectra, gravity classifications were determined on the basis of the relative atomic line ratios. An ellipsis in the gravity column indicates that the $\mathrm{S} / \mathrm{N}$ of the spectra was not sufficient to determine whether absorption lines were present.

e "Possible NIR emission lines" indicates that residual emission lines were present after background sky subtraction, which may originate from the star rather than the nebula. However, because of the very high background of nebular emission, we cannot say with certainty that this is the case. This comment primarily refers to $\mathrm{H}_{2}$ seen in emission. P Cygni profile labels are given as uncertainties for the same reason.

${ }^{\mathrm{f}}$ Optical spectral type from $\mathrm{H} 97$.

g Classification from CRSP spectrum.

${ }^{\mathrm{h}}$ We did not attempt to classify objects earlier than $\mathrm{K} 7$.

$\S 3.1$ ). We did not have this luxury in the $K$ band because of the flat-field issues discussed in $\S 2.3 .1$.

As detailed below, we rely primarily on broadband molecular features for spectral classification. We present our spectral types for objects within the inner $5.1 \times 5$. $^{\prime} 1$ of the $\mathrm{ONC}$ (centered on $\theta^{1} \mathrm{C}$ ) in Table 1 . When possible, objects have been cross-referenced to previous literature, and optical spectral types are given. Table 1 also includes estimates for the surface gravity of each object based on the presence and relative strengths of atomic absorption lines. Most (but not allsee $\S \S 3.1$ and 3.2) of the atomic lines present in main-sequence stars at similar temperatures are much weaker in the ONC spectra because of the young age and consequently lower surface gravity of the star. This effect is enhanced if there is excess continuum emission from a circumstellar disk. Therefore, we do not use the properties of these lines to classify a star except to say that if the lines are present and strong, the star is likely not an ONC member. Our procedures are discussed in $\S \S 3.1$ and 3.2 and result in $\mathrm{FeH}, \mathrm{H}_{2} \mathrm{O}-1$, and $\mathrm{H}_{2} \mathrm{O}-2$ index measurements also listed in Table 1.

\subsection{J-Band Indices}

Figure 3 shows two $J$-band $M$ dwarf standard spectra at different temperatures, taken with NIRSPEC. The strongest atomic line transitions in this wavelength regime are the pairs of $\mathrm{K}_{\mathrm{I}}$ lines at 1.169 and $1.177 \mu \mathrm{m}$ and at 1.243 and $1.252 \mu \mathrm{m}$. As can be seen, the strength of these features increases with later spectral type. This trend is illustrated for a wider range of spectral types in Leggett et al. (1996; stars with spectral types earlier than M7) and McLean et al. (2003; dwarfs of spectral type M6 and later). However, the depth of the lines is also highly dependent on surface gravity. Figure 4 shows four stars of spectral class M7-M8: an optically classified dwarf star (LHS 3003), a newly classified lower surface gravity star of $\sim 600 \mathrm{Myr}$ in Praesepe (RIZ Pr 11), an optically classified star of $\sim 10 \mathrm{Myr}$ in Upper Sco (USCO 128), and a newly classified (see below) Orion star of $\sim 1 \mathrm{Myr}$ (HC 210). This figure illustrates the dramatic decrease in strength of the $\mathrm{K} I$ lines with decreasing surface gravity (see also McGovern et al. 2004; Gorlova et al. 2003). We find these lines to be weak or absent in most of the ONC spectra, confirming cluster membership.

The dominant molecular absorption features are iron hydride $(\mathrm{FeH})$ and water $\left(\mathrm{H}_{2} \mathrm{O}\right)$, the strongest bands of which are found at approximately 1.20 and $1.34 \mu \mathrm{m}$, respectively. Following the work of McLean et al. (2000) and Reid et al. (2001), we have constructed an index, $\mathrm{H}_{2} \mathrm{O}-1$, to measure the strength of the $1.34 \mu \mathrm{m}$ water absorption feature using the 
TABLE 2

Spectral Types for the OUter $^{\mathrm{a}}$ ONC

\begin{tabular}{|c|c|c|c|}
\hline \multirow[b]{2}{*}{$\mathrm{ID}^{\mathrm{b}}$} & \multirow[b]{2}{*}{$K^{\mathrm{b}}$} & \multicolumn{2}{|c|}{ Spectral Type } \\
\hline & & Optical $^{\mathrm{c}}$ & $\mathrm{IR}^{\mathrm{d}}$ \\
\hline 1... & 12.49 & M5. $5^{\mathrm{e}}$ & M6 \\
\hline $205 \ldots \ldots \ldots \ldots \ldots \ldots \ldots \ldots \ldots .$. & 11.68 & $M 6^{\mathrm{e}}$ & M7 \\
\hline 615 & 12.32 & $\mathrm{M} 6^{\mathrm{e}}$ & M7 \\
\hline 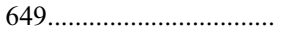 & 12.13 & $\mathrm{M} 6^{\mathrm{e}}$ & M8 \\
\hline 689 & 12.75 & $\mathrm{M} 5.5^{\mathrm{e}}$ & M6 \\
\hline 711 & & $\mathrm{M} 6^{\mathrm{e}}$ & M7: \\
\hline 806 & 12.32 & M5.5 & M6.5 \\
\hline 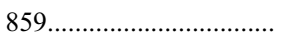 & 11.75 & $\mathrm{M}^{\mathrm{e}}$ & M8 \\
\hline 974 & 11.38 & M5.5 & M6-8 \\
\hline $1036 \ldots \ldots$ & 12.38 & $\mathrm{M} 6^{\mathrm{e}}$ & M6 \\
\hline 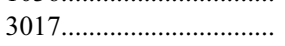 & 12.62 & $\mathrm{M} 4.5^{\mathrm{e}}$ & M2-5 \\
\hline 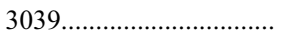 & 11.76 & M5.5 & M7 \\
\hline 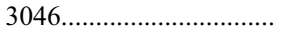 & 11.79 & M5.5 & M5 \\
\hline $5100 \ldots \ldots \ldots \ldots \ldots \ldots$ & 11.81 & $\mathrm{M} 5.5 \mathrm{e}^{\mathrm{e}}$ & M6 \\
\hline
\end{tabular}

a "Outer" in this context refers to the region beyond the $5.1 \times 5$ ' 1 NIRC field centered on $\theta^{1} \mathrm{C}$.

${ }^{\mathrm{b}}$ IDs and $K$ magnitudes from Hillenbrand et al. (1998). See references therein.

${ }^{c}$ Optical spectral types are from LRIS data unless otherwise noted.

${ }_{\mathrm{d}}^{\mathrm{d}}$ Infrared spectral type uncertainties are \pm 2 subclasses unless otherwise noted. A colon indicates that the spectrum had lower $\mathrm{S} / \mathrm{N}$ and that the classification is less certain.

${ }^{\text {e }}$ Optical spectral types come from H97.

ratio of the flux at $1.34 \mu \mathrm{m}$ to that at $1.30 \mu \mathrm{m}$. The positions of the flux bands have been shifted slightly from those of previous authors to avoid contamination by variable emission features that arise in the surrounding nebula and may still be present after sky subtraction in some of the spectra. In addition, we constructed a new index to measure the strength of the $\mathrm{FeH}$ feature using the ratio of flux at $1.20 \mu \mathrm{m}$ to that at $1.23 \mu \mathrm{m}$. Bandwidths are $100 \AA$ for the $\mathrm{H}_{2} \mathrm{O}-1$ index and

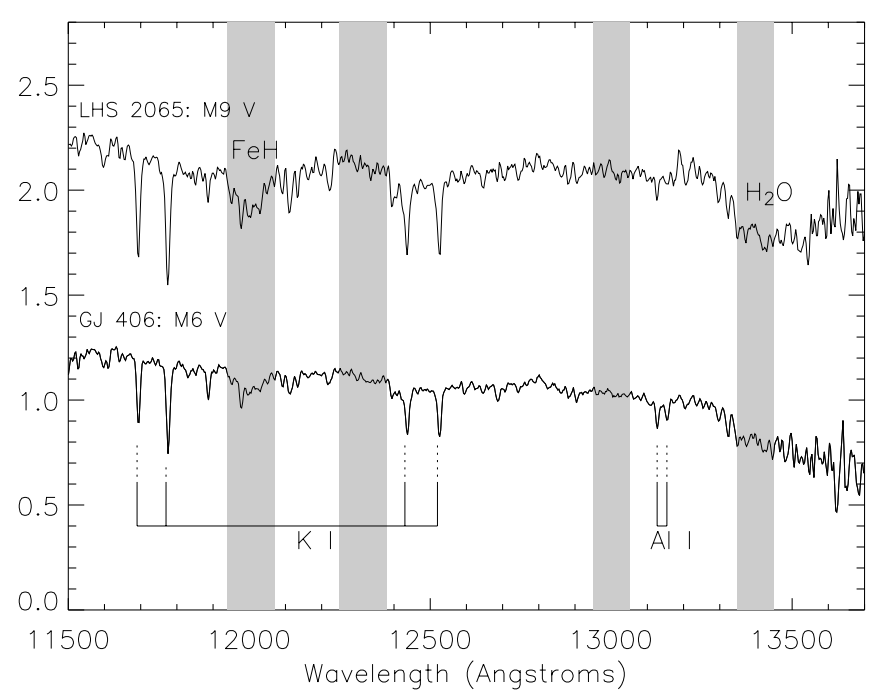

FIG. 3.- J-band NIRSPEC spectra of standard stars at two temperatures. The more prominent atomic (pairs of $\mathrm{K}$ I doublets and an $\mathrm{Al}$ I doublet) and temperature-sensitive molecular features $\left(\mathrm{FeH}\right.$ and $\left.\mathrm{H}_{2} \mathrm{O}\right)$ are labeled. Flux bands centered on molecular and continuum features used in classification are shown as shaded regions.
$130 \AA$ for the $\mathrm{FeH}$ index. The feature and continuum bands are indicated by shaded regions in Figure 3.

We found the water index to be an excellent indicator of spectral type. Figure 5 (top left) shows spectral type versus the $\mathrm{H}_{2} \mathrm{O}-1$ index. Here a spectral type of 0 represents an M0 star, and a spectral type of 10 represents an L0 dwarf. In all panels, filled circles and triangles represent standard-star spectra taken with NIRSPEC at the same time as our program objects. Open circles are the lower resolution data $(R \sim 400)$ taken from the Leggett group. Despite the differences in equipment and data reduction techniques, we find excellent correlation between the two sets of data. The strength of the water index decreases systematically with decreasing spectral type. We derived a functional fit to the combined standard observations, which we used to classify the Orion data. Typical errors are $\sim 1.5$ spectral subtypes. All index fits are given in Table 3.

Figure 5 (top right) shows the spectral type versus the $\mathrm{FeH}$ index. As can be seen, this index works well only for a limited range in spectral type (M3-L3) in which the absorption feature peaks in strength. However, stars earlier than M3 and later than L3 are classifiable by measuring the $\mathrm{H}_{2} \mathrm{O}-1$ index. Furthermore, it is readily apparent via visual inspection of the spectra when a star has been classified by the $\mathrm{FeH}$ index as an M9 but is in fact an L6. Within the spectral range of interest, the strength of the $\mathrm{FeH}$ index decreases systematically with spectral type, similar to the $\mathrm{H}_{2} \mathrm{O}-1$ index. We again derived a functional fit to the combined standard observations to be used in classifying our program objects (Table 3).

In Figure 5 (top left and top right) the filled triangles, which represent the lower surface gravity standard stars, correspond to objects ranging in age from $\sim 600 \mathrm{Myr}$ (Praesepe) to $\sim 10 \mathrm{Myr}$ (Upper Sco and TW Hydrae). As can be seen, we find both molecular temperature (spectral type) indices to be stable to variations in surface gravity. However, great care had to be taken in applying these indices because they are sensitive to the effects of reddening and IR excess (see $\S \S 3.4$ and 3.5). Once preliminary spectral types were derived from both the $\mathrm{H}_{2} \mathrm{O}-1$ and $\mathrm{FeH}$ indices, all objects were inspected

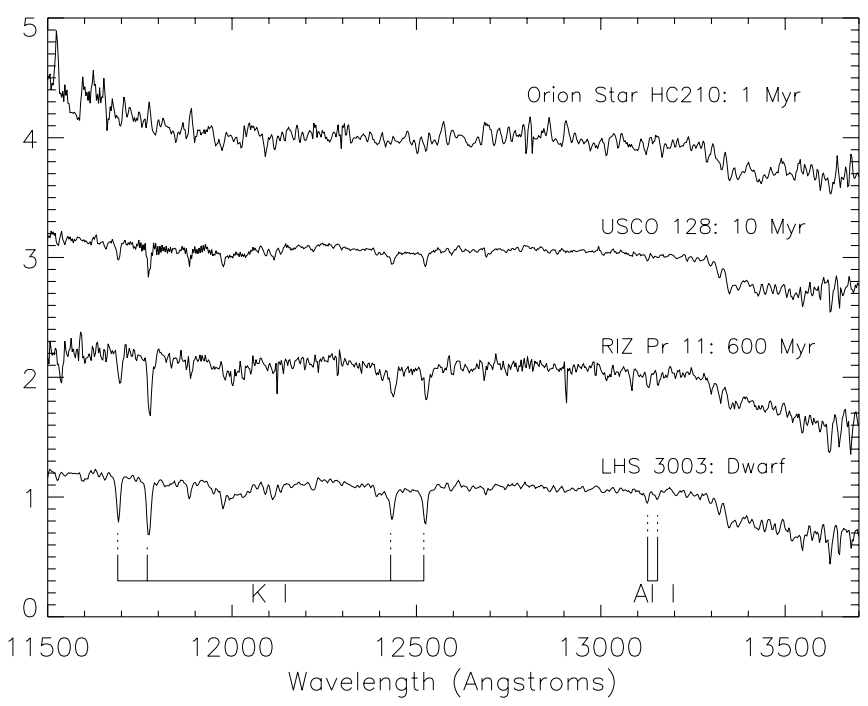

FIG. 4.- $J$-band NIRSPEC data of four spectral class M7-M8 stars: an optically classified main-sequence dwarf star (LHS 3003), a newly classified lower surface gravity star in Praesepe (RIZ Pr 11), an optically classified star in Upper Sco (USCO 128), and a newly classified Orion star (HC 210). Strongly surface gravity-sensitive atomic features $\mathrm{K}$ I and $\mathrm{Al}$ I are labeled. 

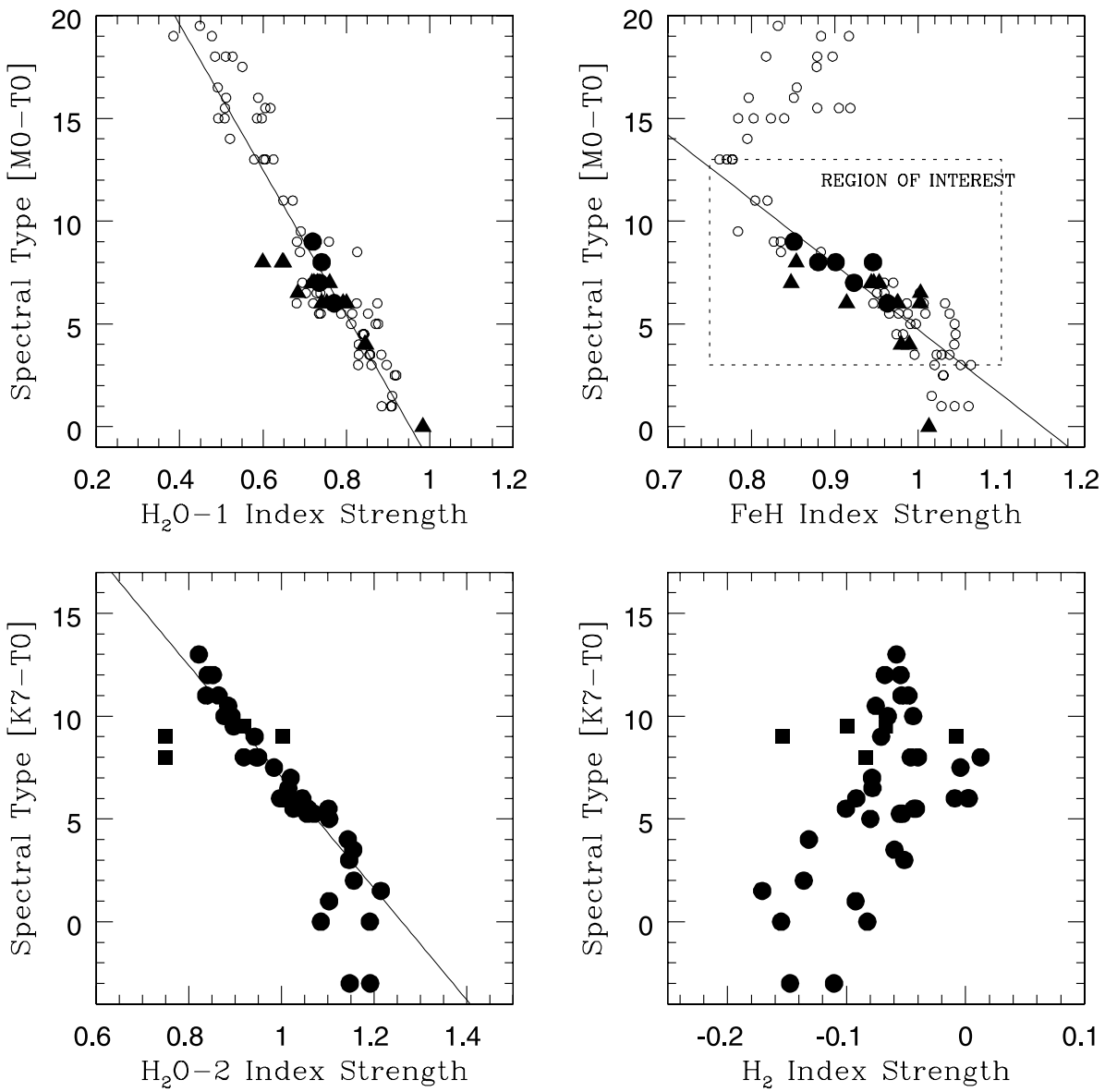

Fig. 5.-Spectral type as a function of classification indices. Filled circles and triangles represent main-sequence standard stars and lower surface gravity PMS standard stars taken with NIRSPEC, respectively. Filled squares correspond to giant stars. Open circles are nearby field star and brown dwarf data from the Leggett group. Solid lines are empirical fits listed in Table 3. The FeH index works well only for a limited range in spectral type (M3-L3) for which the absorption feature peaks in strength. The $\mathrm{H}_{2} \mathrm{O}-2$ index works well for objects later than $\mathrm{M} 2$. We do not derive an empirical fit to the $\mathrm{H}_{2}$ index because its dynamic range is small and the scatter significant. In the spectral type scale used, K7 corresponds to $-3, \mathrm{M} 0$ to $0, \mathrm{~L} 0$ to 10 , and T0 to 20 .

visually and compared with standards for confirmation or minor adjustment.

\subsection{K-Band Indices}

Figure 6 shows a sequence of $K$-band $\mathrm{M}$ and $\mathrm{L}$ dwarf standard spectra taken with NIRSPEC. We find that all the strong atomic line transitions ( $\mathrm{Ca}$ I at $1.978,1.985$, and $1.986 \mu \mathrm{m} ; \mathrm{Al} \mathrm{I}$ at 2.110 and $2.117 \mu \mathrm{m} ; \mathrm{Na} \mathrm{I}$ at 2.206 and $2.208 \mu \mathrm{m}$; and $\mathrm{Ca}$ I at 2.261, 2.263, and $2.266 \mu \mathrm{m}$ ) decrease in strength with temperature. The exceptions are the $\mathrm{Na}$ I lines, which increase in strength until late $M$ stars and then disappear quickly by early L (see also McLean et al. 2000, 2003; Reid et al. 2001). The $1.98 \mu \mathrm{m} \mathrm{Ca}$ I triplet, as well as the $\mathrm{Na}$ I and Al I lines, decreases in strength with decreasing surface gravity but does not disappear completely at early $\mathrm{M}$ spectral types, even in giant stars (Kleinmann \& Hall 1986). The dominant molecular features are $\mathrm{H}_{2} \mathrm{O}(1.90 \mu \mathrm{m}), \mathrm{H}_{2}(2.20 \mu \mathrm{m})$, and $\mathrm{CO}(2.30 \mu \mathrm{m})$.

It is common practice when classifying low-mass stars from $K$-band spectra to use the strength of the $2.30 \mu \mathrm{m} \mathrm{CO}$

TABLE 3

Empirical Relations

\begin{tabular}{|c|c|c|c|}
\hline Fit & Error & Range & Comment \\
\hline Sp type $=(33.71 \pm 0.79)-(35.35 \pm 1.06) *\left(\mathrm{H}_{2} \mathrm{O}-1\right) \ldots \ldots \ldots$ & $\sigma= \pm 1.2$ & M0-T0 & In the spectral type relations for band index fits $\mathrm{M} 0$ is \\
\hline Sp type $=(36.31 \pm 1.46)-(31.59 \pm 1.59) *(\mathrm{FeH}) \ldots \ldots \ldots \ldots$ & $\sigma= \pm 0.66$ & M3-L3 & respresented by $0, \mathrm{M} 5$ by 5 , and $\mathrm{L} 0$ by 10 . All Sp type \\
\hline Sp type $=(34.13 \pm 1.19)-(27.10 \pm 1.20) *\left(\mathrm{H}_{2} \mathrm{O}-2\right) \ldots \ldots \ldots$ & $\sigma= \pm 0.53$ & M2-L3 & equations assume dereddened band index measurements. \\
\hline$T_{e f f}=-224536+111513 *(\mathrm{Sp}$ type $)-$ & & & \\
\hline $\begin{array}{l}17907.9 *(\text { Sp type })^{2}+943.944 *(\text { Sp type })^{3} \ldots \ldots \ldots \ldots \ldots \ldots . . . \\
(H-K)_{o}=51.4776-23.9402 *(\text { Sp type })+\end{array}$ & $\sigma= \pm 33.9$ & K7-L0 & In the spectral type relations for HR diagram transforms \\
\hline $\begin{array}{l}3.67070 *(\mathrm{Sp} \text { type })^{2}-0.184388 *(\mathrm{Sp} \text { type })^{3} \ldots \ldots \ldots \ldots \ldots \ldots \ldots \ldots \ldots \ldots \ldots \ldots \\
\mathrm{BC}_{K}=57.1637-27.7257 *(\mathrm{Sp} \text { type })+\end{array}$ & $\sigma= \pm 0.033$ & K7-L0 & $\mathrm{M} 0$ is represented by 6.0 , M 5 by 6.5 , and $\mathrm{L} 0$ by 7.0 . \\
\hline 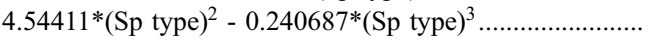 & $\sigma= \pm 0.037$ & K7-L0 & \\
\hline
\end{tabular}




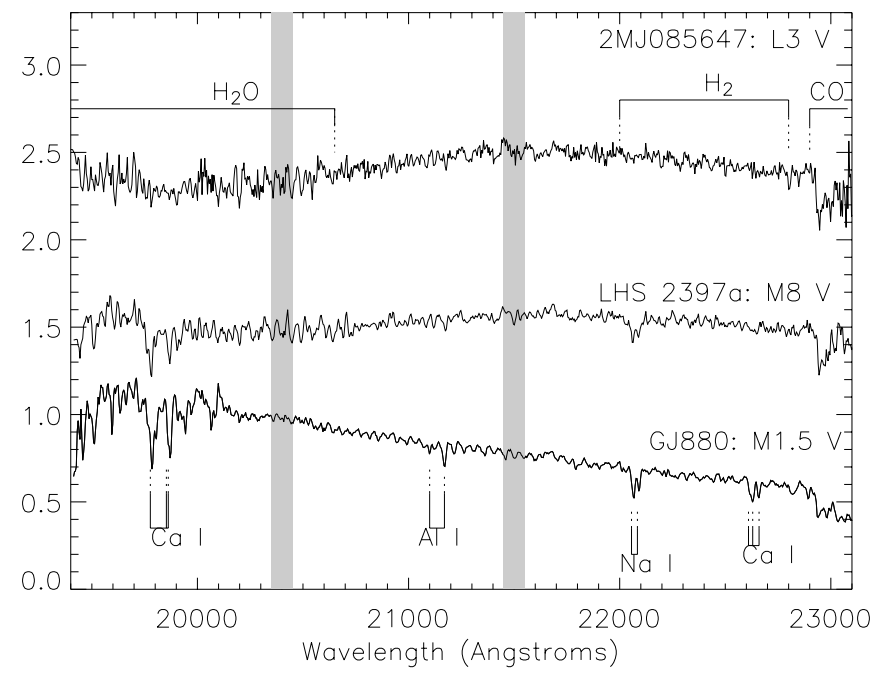

FIG. 6. $-K$-band spectra of standard stars taken over a wide range in temperature. Atomic lines ( $\mathrm{Ca}$ I, $\mathrm{Al}$ I, and $\mathrm{Na}$ I) and molecular features $\left(\mathrm{H}_{2} \mathrm{O}\right.$, $\mathrm{H}_{2}$, and $\mathrm{CO}$ ) are labeled. Flux bands centered on $\mathrm{H}_{2} \mathrm{O}$ and continuum features used in the classification are shown as shaded regions.

absorption feature. Because the $K^{\prime}$ filter used in our NIRSPEC setup begins to cut out at $\lambda \sim 2.295 \mu \mathrm{m}$, we find the depth of this feature in our data to be unreliable and therefore do not use it in classification. Again, following the work of McLean et al. (2000) and Reid et al. (2001), we have constructed an index to measure the strength of the very broad $1.90 \mu \mathrm{m} \mathrm{H}_{2} \mathrm{O}$ feature (which affects the spectra from the short-wavelength end of our spectral range through $\sim 2.1 \mu \mathrm{m}$ ). We define our index, $\mathrm{H}_{2} \mathrm{O}-2$, as the ratio of the flux at $2.04 \mu \mathrm{m}$ to that at $2.15 \mu \mathrm{m}$ (Fig. 6, shaded regions). The indices have been shifted slightly from those of other authors because of ONC nebular emission features. A plot of spectral type versus the $\mathrm{H}_{2} \mathrm{O}-2$ index is shown in Figure 5 (bottom left). The strength of this index decreases with decreasing spectral type, similar to index behavior in the $J$ band. We find good correlation for objects later than $\sim \mathrm{M} 2$. We derived an empirical fit to the standard observations, which we used to classify our program stars. Typical errors are $\sim 1.5$ spectral subtypes. Filled squares in Figure 5 (bottom left and bottom right) correspond to low surface gravity field giant stars. Wilking et al. (2004) found their $K$-band water index (which incorporates both the 1.9 and $2.5 \mu \mathrm{m} \mathrm{H}_{2} \mathrm{O}$ absorption features) to be insensitive to surface gravity in the dwarf to subgiant range. These results are consistent with those of Gorlova et al. (2003), who compared the water index for spectral M-type young cluster objects and a sample of M-type field stars. However, Wilking et al. (2004) do find significant scatter in the measure of this index for giant stars. They attribute this result in part to the variable nature of late-type giants. We draw a similar conclusion for the water index of giant stars from our own admittedly extremely limited sample (of which three out of four are Mira variables). Since we do not have a large grid of young standard stars from which to derive our own assessment of surface gravity effects on $K$-band $\mathrm{H}_{2} \mathrm{O}$ absorption, we assume those of Wilking et al. (2004) and Gorlova et al. (2003) and apply the relationship found for dwarf stars to our sample of PMS targets.

Finally, Tokunaga \& Kobayashi (1999) have shown $\mathrm{H}_{2}$ absorption to be present at $\sim 2.20 \mu \mathrm{m}$ in late $\mathrm{M}$ and early L dwarfs. They define an index,

$$
K 2=\frac{\left\langle F_{2.20-2.28}\right\rangle-\left\langle F_{2.10-2.18}\right\rangle}{0.5\left(\left\langle F_{2.20-2.28}\right\rangle+\left\langle F_{2.10-2.18}\right\rangle\right)},
$$

where $\left\langle F_{\lambda_{1}-\lambda_{2}}\right\rangle$ is the average flux between $\lambda_{1}$ and $\lambda_{2}$. We use a similar index, $\mathrm{H}_{2}$, using the integrated flux between $\lambda_{1}$ and $\lambda_{2}$. A plot of spectral type versus $\mathrm{H}_{2}$ is shown in Figure 5 (bottom right). Because the dynamic range of this index is small and the scatter significant, we do not derive an empirical fit. However, the presence of $\mathrm{H}_{2}$ absorption is readily detected in spectra of late $M$ and early $L$ objects through visual inspection and was used in classification. For reference, we have included measurements of $\mathrm{H}_{2}$ in Table 1 .

\subsection{CRSP Spectra}

All $J$ - and $K$-band ONC spectra taken with CRSP were classified using the spectral features discussed above. Differences in equipment and data reduction techniques caused an offset in the continuum shape compared with the NIRSPEC data, and therefore, we could not use the same band index relations. Because we had CRSP spectra for only 16 ONC objects, we relied on visual inspection for classification. Extinction estimates were made in the same manner as for the NIRSPEC data (see $\S 3.4$ ), and object spectra were compared with artificially reddened spectra of standard stars taken with CRSP along with the target objects. Spectral types for objects inside and outside the inner 5.'1 $\times 5$ ' 1 of the ONC are given in Tables 1 and 2, respectively. All objects have optical classifications either in the literature or from new LRIS spectra, and these are also listed.

\subsection{Extinction}

As mentioned in the previous sections, the indices we use to derive spectral types are sensitive to effects from extinction, which differentially reddens the spectra, thus changing the band-to-continuum ratios. Figure 7 shows an M9 mainsequence standard star (LHS 2065) that was observed during both NIRSPEC observing runs ( $J$ and $K$ band). In each panel, the top spectrum represents the original data. The subsequent spectra have been artificially reddened by 5,10 , and $15 \mathrm{mag}$ of extinction using the IRAF DEREDDEN package (which incorporates the empirical selective extinction function of Cardelli et al. 1989). The flux bands for the classification indices described in $\S 3.1$ are shown as shaded regions, and the $K$-band $\mathrm{H}_{2}$ absorption region is marked. We expect a systematic shift in all the indices with increased reddening.

To determine the magnitude of this effect, we artificially reddened all standard objects by 1-20 mag and remeasured each band index. We then derived empirical fits of the shift of each classification index as a function of increasing extinction. Figure 8 illustrates the resulting spectral type shift plotted as a function of $A_{V}$ for all three indices. Extinction causes us to systematically classify a star later than it is if we use the $\mathrm{FeH}$ and $\mathrm{H}_{2} \mathrm{O}-2$ indices and earlier than it is if we use the $\mathrm{H}_{2} \mathrm{O}-1$ index. Error bars correspond to errors in the fit, which increase with increasing $A_{V}$. As can be seen, extinction must be taken into consideration when applying the indices. We find an average value of $A_{V} \sim 6$ for our objects, which would result in a spectral type shift of approximately \pm 2 subtypes for all indices if not accounted for.

We use color information to derive an initial extinction estimate for each of our spectral sources. It has been shown (see Hillenbrand et al. 1998; Lada et al. 2000; Muench et al. 2001 ) that $\sim 50 \%$ of sources in the ONC show signs of $J-H$ 

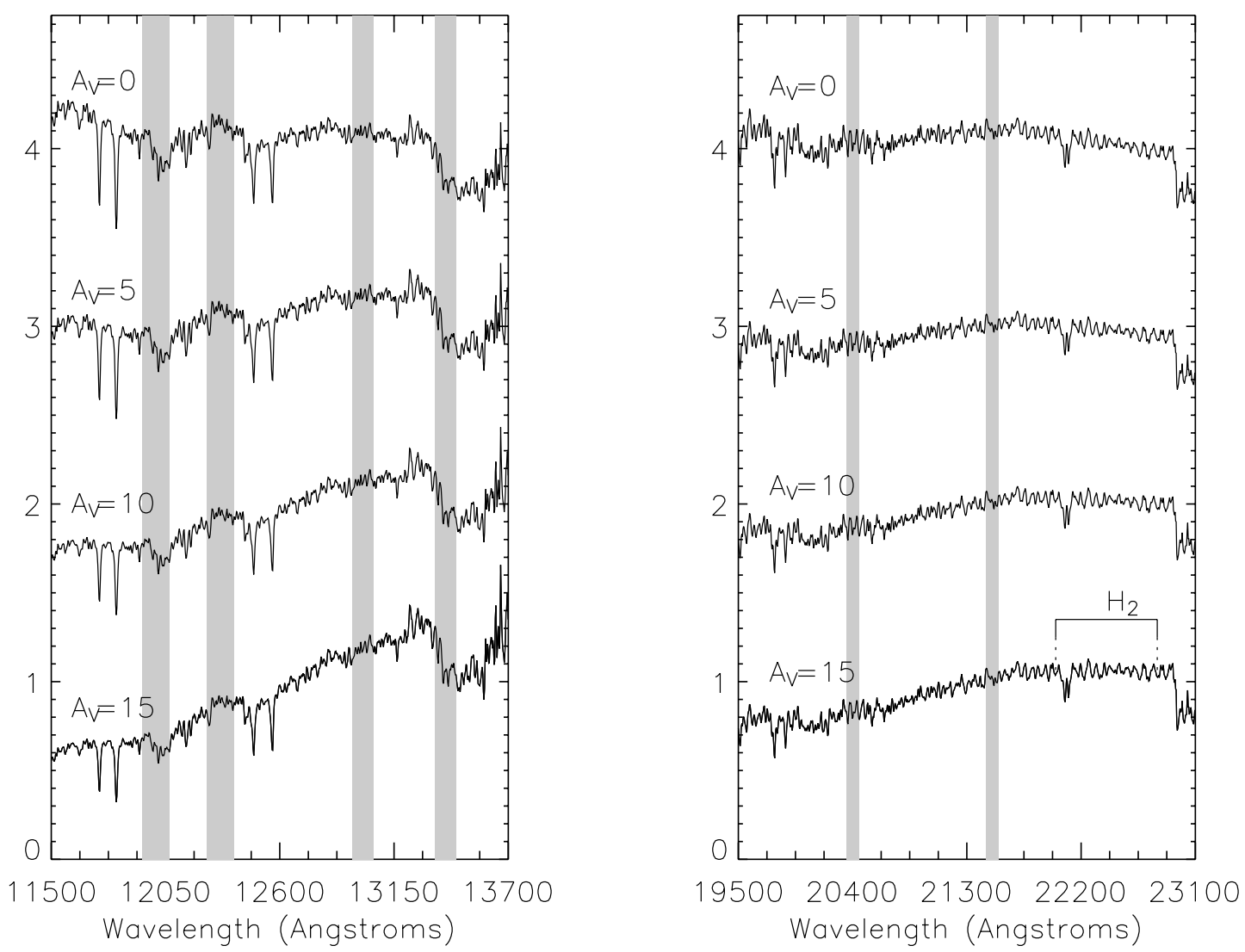

FIG. 7.-M9 main-sequence standard star (LHS 2065) that was observed during both NIRSPEC observing runs ( $K$ and $J$ band). In each panel, the top spectrum shows the original data. Subsequent spectra have been artificially reddened by 5,10 , and 15 mag of visual extinction. The flux bands corresponding to classification

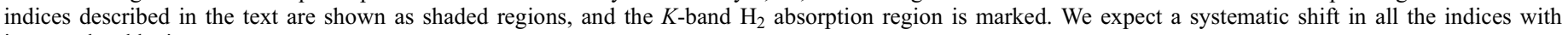
increased reddening.

and $H-K$ infrared excess consistent with emission from a circumstellar disk. Assuming the $H-K$ excess to arise from extinction alone without taking into account infrared excess results in overestimates of $A_{V}$. Meyer et al. (1997) showed that the intrinsic colors of late $\mathrm{K}$ and early $\mathrm{M}$ stars with disks are confined to a classical T Tauri (CTTS) star locus in the $(J-H),(H-K)$ color-color plane. Following the work of M02, we derive individual reddening estimates for each star by dereddening its $J-H$ and $H-K$ colors back to this CTTS locus. Stars falling below this locus (primarily because of photometric scatter) are assumed to have $A_{V}=0$. For 12 of the objects that have no $J$ magnitudes available, $A_{V}$ values were estimated by dereddening $K$ and $H-K$ magnitudes and colors back to a theoretical $1 \mathrm{Myr}$ isochrone.

Because the $\mathrm{HCOO}$ data set does not include $J$-band photometry, we used the color data of M02 for dereddening purposes. In comparing the two photometric data sets, M02 and $\mathrm{HC} 00$, we derived $1 \sigma$ standard deviations of $\sim 0.35$ for the $H$ and $K$ magnitudes. The scatter appears to be random for the $K$ magnitudes, but the $H$ photometry of M02 is systematically brighter than the photometry of HCO0. Scatter between the two data sets was also noted by M02, who attributed
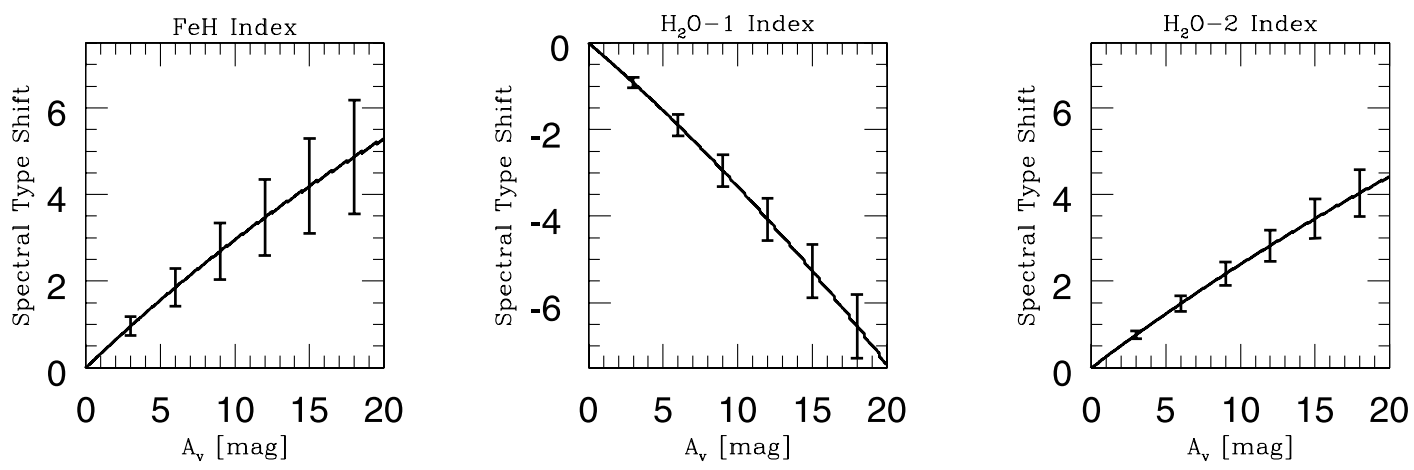

FIG. 8.- Spectral type shift produced from index measurements as a function of $A_{V}$. Extinction causes us to systematically classify a star as later than it is by

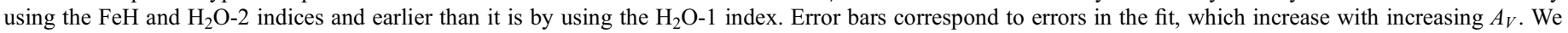

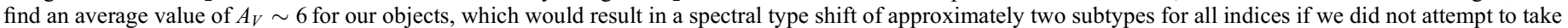
extinction into account during the classification process. 


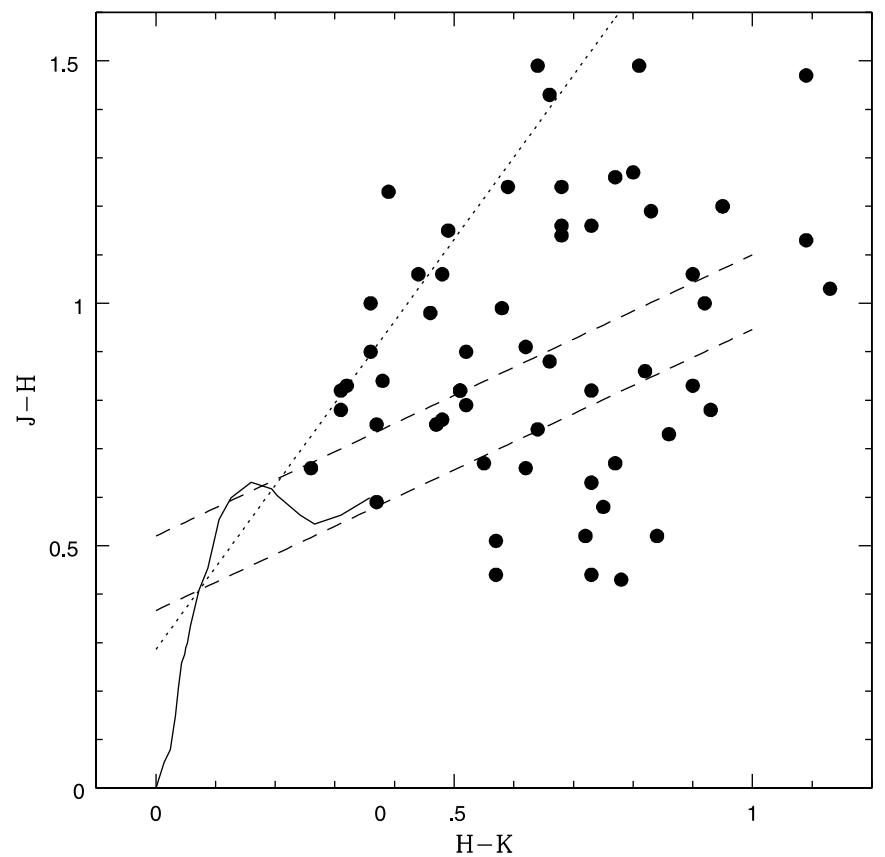

FIG. 9.-Color-color diagram of ONC stars for which we have new nearinfrared spectral types later than K7. Data are taken from M02. The solid line represents the intrinsic colors of main-sequence dwarf stars as given by Bessell \& Brett (1988), transformed to the CIT photometric system. The slope of the interstellar reddening vector (dotted line) is that of Cohen et al. (1981). Dashed lines indicate the upper and lower boundaries of the CTTS locus (Meyer et al. 1997), shifted to apply to K7-L3 dwarfs. Note that we have not accounted for the intrinsic width of the locus as it is defined or for any possible change in slope of the locus with later spectral types. If attributed to reddening, the width of the region corresponds to $\Delta A_{V} \sim 2$, or less than one spectral subtype (see Fig. 8).

the difference in part to the intrinsic infrared variability of low-mass PMS stars. Variables in the Orion A molecular cloud have been found to have an average amplitude change of $\sim 0.2 \mathrm{mag}$ at near-infrared wavelengths on weekly to monthly scales (Carpenter et al. 2001). M02 also noted that because of the strong nebular background, differences in aperture sizes contribute to photometric noise.

For each program object we apply the empirical fits shown in Figure 8 to deredden the measured spectral typing indices and then use the new value to derive an estimate of spectral type. We compare each spectrum with standard stars, artificially reddened to the same extinction value. All spectral types were verified visually to avoid contamination of band index measurements arising from noise in the spectra.

A possible bias in the above analysis is that the CTTS locus of Meyer et al. (1997) applies to the Taurus cluster, where most of the stars have spectral types K7-M0. When applying the locus to stars outside this spectral range, we must take into account its anticipated width. Figure 9 is a $(J-H),(H-K)$ CMD for all our ONC stars with spectral types (excepting 12 stars for which there were no $J$-band data available). The top dashed line is the CTTS locus for Taurus stars, which defines an upper bound to the locus when we consider all stars with spectral types K7-L3. The bottom dashed line defines a lower bound to this locus. The width of the region corresponds to a $\Delta A_{V}$ of $\sim 2$, or $\sim 0.5$ spectral subtype uncertainty (see Fig. 8). Since intrinsic spectral type errors derived from the indices are $\approx 1.5-2$ subtypes, ignoring this effect does not result in significant biases. In referring to the width of the locus, we have considered only how the CTTS line as defined by Meyer et al.
(1997) would shift if it applied to stars with K7-L3 spectral types; we have not accounted for the intrinsic width of the locus as it is defined nor for any possible shift in slope of the locus with spectral type. We emphasize that we are using this method to derive an $A_{V}$ estimate only, to aid us in spectral classification. To place a star on the H-R diagram, more precise extinction values are derived by combining a star's photometric and spectroscopic data (see $\S 4.1$ ).

\subsection{Infrared Excess}

As mentioned previously, we expect $\sim 50 \%$ of the ONC sources to have a wavelength-dependent infrared excess due to thermal emission from warm dust grains in a circumstellar disk. This excess dilutes (veils) the strength of molecular absorption features used in spectral classification. From experiments with artificially veiled standard stars, we find that veiling can make a later type spectrum look like an earlier type photosphere. However, since the photospheric flux of late-type stars peaks at $1-1.5 \mu \mathrm{m}$, we do not expect extremely large excesses at near-infrared wavelengths. The veiling index is defined as $r_{\lambda}=F_{\lambda \text { ex }} / F_{\lambda \text { ph }}$, where "ex" indicates "excess" and "ph" indicates "photosphere." Meyer et al. (1997) found that K7-M0 CTTSs have a median veiling value of $r_{K} \approx 0.6$. We expect this value to be lower for cooler objects whose photospheric emission peaks at longer near-infrared wavelengths.

Figure 10 shows the expected color excess for stars of varying spectral type veiled by a $T=1500 \mathrm{~K}$ blackbody. Data points are labeled by spectral type, and the two rows correspond to $r_{K}=0.5$ (bottom row) and $r_{K}=1$ (top row). As can be seen, for $r_{K}<0.5$, we expect color excesses $\Delta(H-K)$ and $\Delta(J-H) \lesssim 0.2 \mathrm{mag}$. The effect on classification is $\approx 1.5-2$ spectral subclasses earlier or later for the $\mathrm{H}_{2} \mathrm{O}-2$ and $\mathrm{FeH} / \mathrm{H}_{2} \mathrm{O}-1$ indices. This result is somewhat overestimated for later spectral types (cooler than $\sim \mathrm{M} 6$ ) with $K$-band spectra for which it is more readily apparent via visual inspection whether veiling is affecting spectral classification. Because we have no way to independently measure veiling in our spectra (modeling of high-dispersion data is required), we simply note the possible bias.

\subsection{Summary of Spectral Classification}

In all cases, classification was performed first using flux ratios of broad molecular absorption lines to continuum, then comparing visually with standards. Extinction was estimated assuming CTTS colors and taken into account during the classification process. The influence of veiling was investigated but not explicitly accounted for during spectral classification. Surface gravity was assessed visually from the presence or strength of atomic absorption lines. For $J$-band spectra an object was given a gravity classification of "low" if it had no detectable atomic absorption lines and "high" if it had strong lines, similar to those seen in spectra of dwarf standards of the same spectral type. A classification of "int" indicates that absorption lines were present but were not as strong as those in dwarf stars at the same temperatures (see Table 1 ). For $K$-band spectra gravity determinations were given based on the relative strength of atomic absorption features.

From the NIRSPEC data, we have classified 71 objects in the inner $\mathrm{ONC}$ to be of spectral type $\mathrm{K} 7$ or later, $\approx 50 \%$ of which are M6 or later. At an age of 1-2 Myr, all objects with spectral types later than M6 are substellar $\left(M<0.08 M_{\odot}\right)$ on the basis of comparison with theoretical models (e.g., DM97). In addition, we have classified 16 spectra of objects in the 

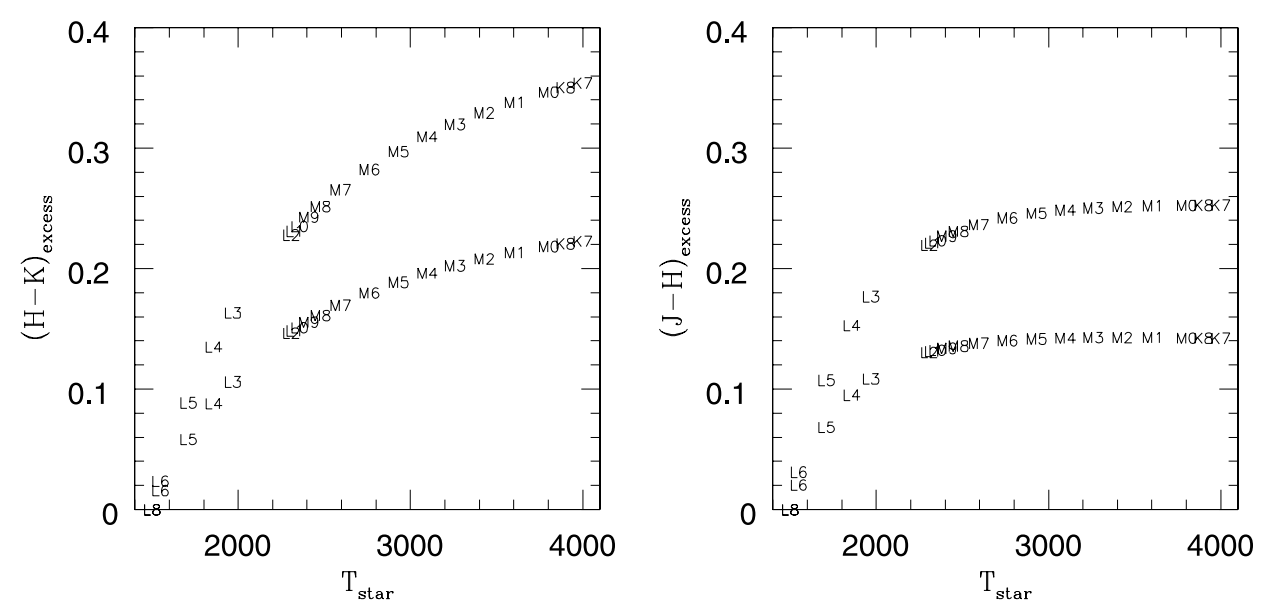

FIG. 10.-Expected color excess for low-mass stars veiled by a $T=1500 \mathrm{~K}$ blackbody. Data points are labeled by spectral type, and the two rows correspond to $r_{K}=0.5$ (bottom row) and 1 (top row). For $r_{K}<0.5$, we expect color excesses $\Delta(H-K)$ and $\Delta(J-H) \lesssim 0.2$ mag. The effect of veiling on classification is $\approx 1.5-2$ spectral subclasses earlier for the $\mathrm{H}_{2} \mathrm{O}-2$ and $\mathrm{FeH}$ indices and later for the $\mathrm{H}_{2} \mathrm{O}-1$ index.

inner and outer parts of the nebula taken with CRSP, nine of which are M6 or later. Finally, we also report in Tables 1 and 2 new optical spectral types obtained with LRIS, as well as those reported previously in the literature. For the large majority of sources there is excellent agreement (to fewer than two spectral subclasses) between optical and infrared spectral types.

\section{H-R DIAGRAM}

In this section we combine each object's spectral type and near-infrared photometry to derive values for its luminosity and effective temperature and place it on the theoretical H-R diagram. The goal is to transform temperatures and luminosities into ages and masses using theoretical PMS evolutionary tracks. From this information we can explore the ONCs IMF down to less than $0.02 M_{\odot}$ using spectroscopically confirmed cluster members.

\subsection{Effective Temperatures, Intrinsic Colors, and Bolometric Luminosities}

Spectral types of K7-L3 were converted to temperatures based on the scales of Wilking et al. (1999), Reid et al. (1999), and Bessell (1991). An empirical fit for $\mathrm{BC}_{K}$ was determined from the observational data of Leggett et al. (1996, 2002; spectral types M1-M6.5 and M6-L3). We determined intrinsic colors by computing empirical fits to a combination of Bessell \& Brett (1988) theoretical models (for spectral types K7-M6) and Dahn et al. (2002) observed data (spectral types M3.5-L3). $H-K$ color excesses were converted to $A_{K}$ using the reddening law of Cohen et al. (1981).

Because $J$ magnitudes are not available for all program stars, for consistency we used $H-K$ colors and $K$ magnitudes from $\mathrm{HCOO}$ to determine extinction values and estimate bolometric corrections. Using $H-K$ colors rather than $J-H$ colors to predict extinction results in higher derived $A_{V}$ estimates by an average of $\sim 3$ mag for our data $\left(\sim 0.2 \mathrm{mag}\right.$ in $\left.A_{K}\right)$. In addition, if the object has substantial infrared excess, its $K$-band magnitude will be brighter than the photosphere. However, the combination of these effects does not produce a significant shift in placement of a star on the H-R diagram. We find an average difference of $\Delta \log L \approx 0.05$ between luminosities derived using $J$ and $J-H$ versus $K$ and $\mathrm{H}-\mathrm{K}$ for objects with both $J$ - and $K$-band data available. A similar trend of comparable magnitude results if we compare $A_{V}$ values derived from $H-K$ colors alone rather than using both $J-H$ and $H-K$ to deredden stars back to the CTTS locus (see $\S 3.4)$. The uncertainty in derived $A_{V}$ for an M6 star with large color and spectral type uncertainties $\left(\sigma_{H-K} \sim 0.05\right.$ mag and $\sigma_{\text {SpT }} \sim 2$ subclasses, where "SpT" indicates "spectral type") is $\approx 1.5$ mag. All fits are given in Table 3 , and derived quantities for stars included in the H-R diagram are given in Table 4.

For all derived quantities discussed above, we used dwarf scales despite the lower surface gravity of young PMS stars. It has been known since the earliest work on T Tauri stars (Joy 1949) that young PMS objects are observationally much closer to dwarfs than to giants or even subgiants. Since to date there is no accurate temperature or bolometric correction scale for PMS stars, we use the higher gravity dwarf scales in our analysis.

\subsection{H-R Diagram for Objects with NIRSPEC Data}

In Figure 11 we present an H-R diagram for those objects within the inner 5.'1 $\times 5$ !' 1 of the ONC (survey area of HC00) for which we have new spectral types. Objects that were classified using spectra from different instruments are plotted as different symbols. Surface gravity assessment is indicated, and a typical error bar for an M6 \pm 1.5 star is shown in the lower left corner. The PMS model tracks and isochrones of DM97 are also shown. Figure 11 illustrates that we are able to probe lower masses than previous spectroscopic studies have, down to $0.02 M_{\odot}$.

From examination of Figure 11, it appears that we are exploring two separate populations: the inner ONC at $\lesssim 1 \mathrm{Myr}$, and an older population at $\sim 10$ Myr. None of the members of this apparently older population have gravity features that indicate that they might be foreground M stars. Spectra of these objects indicate instead that they have low surface gravity consistent with young objects and are therefore also candidate cluster members. For the remainder of $\S 4.2$ we discuss the possible explanations for this surprising population. First, we detail possible systematic errors in the data reduction and analysis that could in principle cause one to "create" older stars in the H-R diagram. Next, we discuss their possible origin if they are a real feature, and finally, we 
TABLE 4

Derived Quantities for Stars in the H-R Diagram

\begin{tabular}{|c|c|c|c|c|}
\hline $\mathrm{ID}^{\mathrm{a}}$ & $\begin{array}{c}A_{V} \\
(\mathrm{mag})\end{array}$ & $\log \left(T_{\text {eff }} / \mathrm{K}\right)$ & $\log \left(L / L_{\odot}\right)$ & $\begin{array}{c}M \\
\left(M_{\odot}\right)\end{array}$ \\
\hline 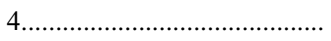 & 4.67 & 3.453 & -1.686 & 0.08 \\
\hline 15 & 8.47 & 3.502 & -1.662 & 0.19 \\
\hline 20 & 3.77 & 3.397 & -1.507 & 0.04 \\
\hline $22 \ldots \ldots$ & 1.78 & 3.397 & -1.178 & 0.05 \\
\hline 25 & 6.00 & 3.556 & -1.080 & 0.42 \\
\hline $27 \ldots \ldots \ldots$ & 9.00 & 3.465 & -2.072 & 0.08 \\
\hline 29 & 4.77 & 3.465 & -0.400 & 0.11 \\
\hline 30 & 2.18 & 3.535 & -1.713 & 0.31 \\
\hline 35 & 1.94 & 3.418 & -0.315 & 0.10 \\
\hline 48 & 5.37 & 3.490 & -1.876 & 0.15 \\
\hline 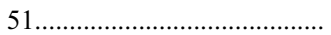 & 2.26 & 3.465 & -0.447 & 0.11 \\
\hline 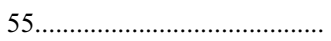 & 1.90 & 3.397 & -1.540 & 0.03 \\
\hline 59. & 0.02 & 3.556 & -2.251 & 0.34 \\
\hline $62 \ldots \ldots \ldots$ & 5.85 & 3.380 & -1.920 & 0.02 \\
\hline 64 & 4.46 & 3.397 & -1.617 & 0.03 \\
\hline 70 (2) & 9.48 & 3.380 & -1.953 & 0.02 \\
\hline 90 & 6.77 & 3.407 & -0.915 & 0.07 \\
\hline 93................................. & 8.24 & 3.513 & 0.692 & 0.18 \\
\hline $111 \ldots$ & 8.61 & 3.380 & -1.837 & 0.03 \\
\hline 114 & 1.44 & 3.418 & -1.464 & 0.05 \\
\hline $123 \ldots \ldots \ldots$ & 7.89 & 3.407 & -1.515 & 0.04 \\
\hline $127 \ldots \ldots$ & 12.51 & 3.574 & -1.072 & 0.58 \\
\hline $143 \ldots \ldots$ & 6.31 & 3.525 & -1.560 & 0.27 \\
\hline $162 \ldots \ldots \ldots$ & 9.17 & 3.453 & -0.553 & 0.11 \\
\hline 167 & 9.19 & 3.407 & -1.631 & 0.03 \\
\hline 200 & 4.58 & 3.513 & -1.838 & 0.22 \\
\hline 210 & 4.81 & 3.418 & -1.636 & 0.04 \\
\hline 212 & 3.41 & 3.380 & -1.559 & 0.03 \\
\hline $221 \ldots \ldots \ldots$ & 4.43 & 3.407 & -1.577 & 0.04 \\
\hline $237 \ldots \ldots \ldots$ & 1.44 & 3.535 & -1.097 & 0.29 \\
\hline 264 & 1.67 & 3.441 & -0.810 & 0.10 \\
\hline 288 & 4.08 & 3.556 & 0.161 & 0.23 \\
\hline $290 \ldots \ldots \ldots$ & 6.64 & 3.453 & -1.039 & 0.11 \\
\hline 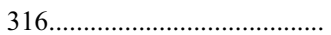 & 10.89 & 3.483 & -1.529 & 0.15 \\
\hline $346 \ldots \ldots \ldots \ldots-1$ & 5.81 & 3.535 & 0.247 & 0.18 \\
\hline 355 & 3.32 & 3.465 & -0.695 & 0.12 \\
\hline 365 & 8.23 & 3.418 & -1.804 & 0.04 \\
\hline 366 & 10.25 & 3.407 & -0.657 & 0.08 \\
\hline 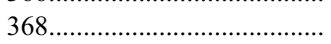 & 8.77 & 3.465 & 0.014 & 0.10 \\
\hline $372 \ldots \ldots$ & 2.04 & 3.380 & -1.711 & 0.03 \\
\hline $381 \ldots \ldots$ & 3.93 & 3.519 & -0.079 & 0.17 \\
\hline 383 & 9.35 & 3.490 & -1.247 & 0.16 \\
\hline 395 & 12.41 & 3.535 & 0.744 & 0.20 \\
\hline 396. & 13.53 & 3.441 & -0.193 & 0.10 \\
\hline $400 \ldots \ldots \ldots$ & 2.16 & 3.380 & -1.978 & 0.02 \\
\hline 403 & 6.78 & 3.418 & -1.754 & 0.04 \\
\hline 409...................................... & 12.51 & 3.574 & -1.010 & 0.59 \\
\hline $429 \ldots \ldots \ldots$ & 5.53 & 3.407 & -1.798 & 0.03 \\
\hline 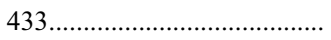 & 10.44 & 3.397 & -1.823 & 0.02 \\
\hline $434^{\mathrm{a}}$ & 21.41 & 3.535 & 0.983 & 0.20 \\
\hline $454 \ldots \ldots$ & 7.85 & 3.535 & -0.314 & 0.23 \\
\hline 455 & 8.21 & 3.490 & -1.881 & 0.15 \\
\hline 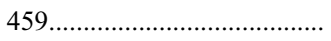 & 1.59 & 3.441 & -0.786 & 0.10 \\
\hline 467................................. & 2.55 & 3.441 & -0.284 & 0.10 \\
\hline 509 & 4.86 & 3.477 & -1.813 & 0.12 \\
\hline 515 & 23.29 & 3.418 & -1.452 & 0.05 \\
\hline 529 & 25.18 & 3.397 & -1.530 & 0.03 \\
\hline 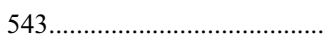 & 4.00 & 3.556 & 0.299 & 0.21 \\
\hline 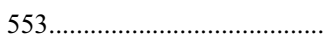 & 5.77 & 3.465 & -0.592 & 0.11 \\
\hline 555 & 1.56 & 3.441 & -0.482 & 0.10 \\
\hline 559 & 0.00 & 3.397 & -1.729 & 0.03 \\
\hline 565 & 5.60 & 3.397 & -1.764 & 0.02 \\
\hline 594 & 2.20 & 3.407 & -1.707 & 0.03 \\
\hline 600 & 14.26 & 3.465 & -0.263 & 0.11 \\
\hline
\end{tabular}


TABLE 4 -Continued

\begin{tabular}{|c|c|c|c|c|}
\hline $\mathrm{ID}^{\mathrm{a}}$ & $\begin{array}{c}A_{V} \\
(\mathrm{mag})\end{array}$ & $\log \left(T_{\text {eff }} / \mathrm{K}\right)$ & $\log \left(L / L_{\odot}\right)$ & $\begin{array}{c}M \\
\left(M_{\odot}\right)\end{array}$ \\
\hline $684 \ldots \ldots \ldots \ldots \ldots$ & 7.93 & 3.535 & -1.349 & 0.31 \\
\hline 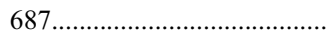 & 6.68 & 3.535 & -1.641 & 0.31 \\
\hline 708 & 28.72 & 3.490 & -0.822 & 0.15 \\
\hline 709 & 2.11 & 3.465 & -2.223 & 0.07 \\
\hline 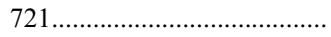 & 9.38 & 3.502 & -1.558 & 0.19 \\
\hline $722 \ldots \ldots \ldots \ldots \ldots$ & 0.00 & 3.369 & -2.989 & 0.02 \\
\hline 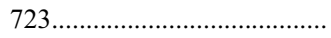 & 3.55 & 3.513 & -2.106 & 0.21 \\
\hline 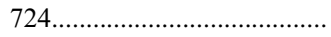 & 0.00 & 3.441 & -2.511 & 0.03 \\
\hline 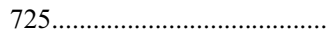 & 9.40 & 3.418 & -2.030 & 0.03 \\
\hline $728 \ldots \ldots \ldots \ldots$ & 5.91 & 3.453 & -2.213 & 0.05 \\
\hline 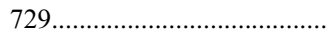 & 14.73 & 3.418 & -1.818 & 0.04 \\
\hline 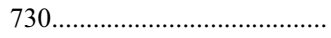 & 15.69 & 3.490 & -1.335 & 0.17 \\
\hline 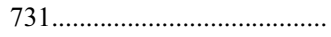 & 19.53 & 3.610 & -1.228 & 0.56 \\
\hline $732 \ldots \ldots \ldots \ldots \ldots \ldots \ldots \ldots$ & 0.00 & 3.525 & -2.508 & 0.23 \\
\hline 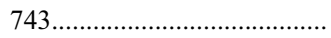 & 5.61 & 3.441 & -2.462 & 0.03 \\
\hline 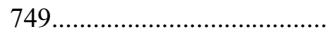 & 3.10 & 3.397 & -2.815 & 0.02 \\
\hline 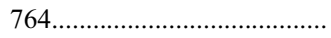 & 7.06 & 3.407 & -1.548 & 0.04 \\
\hline
\end{tabular}

a On the basis of visual inspection of the spectra of $\mathrm{HC} 434$, we find the extinction value derived for this source using the intrinsic colors of a spectral type M2 star to be an overestimate. Instead we use the extinction derived by dereddening its $J-H$ and $H-K$ colors back to the CTTS locus (see $\S 3.4$ ) to determine the derived quantities listed in the table, as well to place HC 434 on the H-R diagram (Fig. 11).

determine why they may not have been detected in previous studies.

There are two possible reasons that we might erroneously detect a bifurcation of the H-R diagram: either the spectral

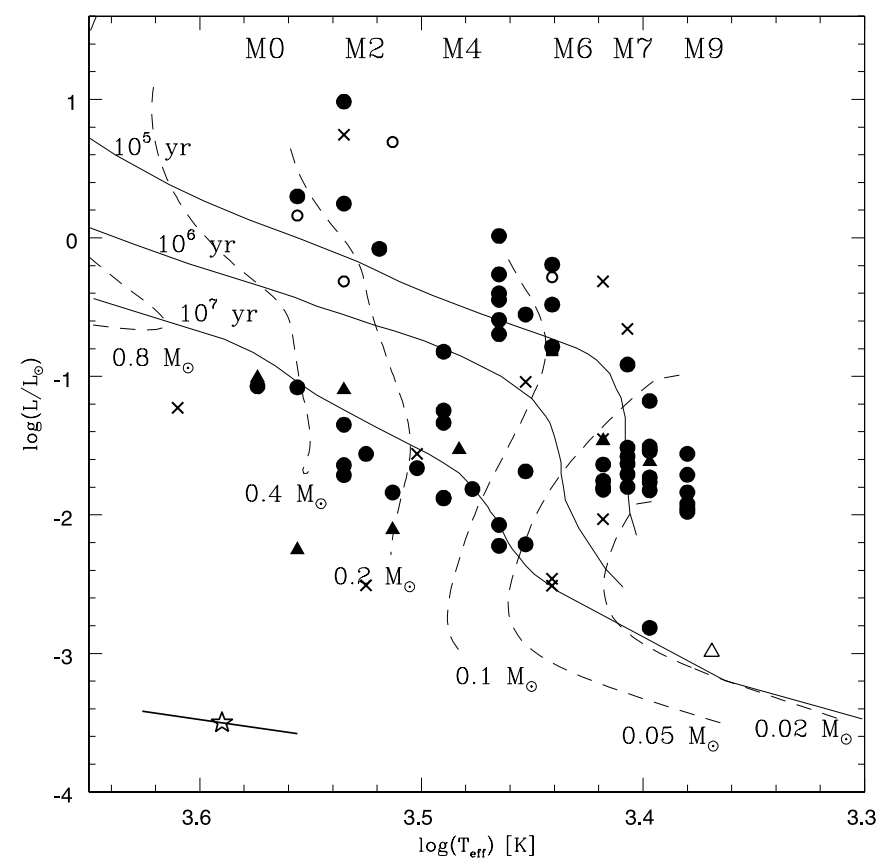

FIG. 11.-H-R diagram of objects within the inner $5.1 \times 5{ }^{\prime} 1$ of the ONC (survey area of $\mathrm{HC} 00$ ) for which we have new spectral types. Circles correspond to stars classified using NIRSPEC and CRSP infrared observations that have good spectral types. Objects with less certain spectral types (those designated with a colon in Table 1) are marked as crosses. Triangles represent objects that have new optical spectral types taken with LRIS. Objects that were classified as "low" and "high" gravity are marked with filled and open symbols, respectively. A typical error bar for an M6 \pm 1.5 star is shown in the lower left corner. The PMS model tracks and isochrones of DM97 are also shown. types are in error and the objects are actually cooler than they are shown to be here or their luminosities have been underestimated. The photometric uncertainties for these objects are less than $0.2 \mathrm{mag}$ for both $H$ and $K$ in the $\mathrm{HC} 00$ data and less than 0.4 mag for $J, H$, and $K$ in the M02 data. The two data sets agree to within $0.8 \mathrm{mag}$ for all objects, and variability, if present, is expected to be $\sim 0.2 \mathrm{mag}$ (see $\S 3.4$ ). An object with spectral type M2 would need to be $\sim 3$ mag brighter in $K$ or its extinction must be underestimated by more than 30 visual mag to move on the H-R diagram from the $10 \mathrm{Myr}$ isochrone to the 1 Myr isochrone. Even when the above uncertainties and possible variability are taken into account, errors of this order are not possible. Moreover, if the photometry were in error, we would expect the result to be continuous scatter in the H-R diagram rather than two distinguishable branches.

The other way to account for the apparently older population through error is if the temperature estimates are too high, i.e., if the spectral types are too early, in some cases by as much as seven spectral subclasses. All spectra have been checked visually by multiple authors (C. L. S and L. A. H.) to ensure accurate classification, and we believe our spectral types are robust to within the errors given. None of the possible biases resulting from assumptions made regarding veiling, extinction, or gravity can have a large enough effect to produce a spectral type offset of this magnitude. One possible exception is that a mid-M (M5) $K$-band spectrum with high reddening can have the same appearance and band index measurements as a later M (M7) spectra with intermediate reddening. But this phenomenon could affect only a small subset of the lower branch population. Therefore, while it may reduce slightly the number of apparently older objects, it cannot correct for them entirely.

The accuracy of our spectral types is supported by the excellent agreement of our near-infrared spectral classifications with existing optical spectral types (most agree to within two spectral subclasses; see Table 1). We find that a significant fraction of the spectra taken with LRIS (Fig. 11, 
filled triangles), which were classified in a manner completely independent of the NIRSPEC and CRSP data, also resulted in apparently $10 \mathrm{Myr}$ old stars. If the older population arises from systematic errors in the reduction or spectral classification of our infrared spectra, we would not expect to find stars classified optically to fall on the same place in the H-R diagram.

If we are to accept the bifurcation as a real feature in the $\mathrm{H}-\mathrm{R}$ diagram, several scenarios could in principle account for it. The first is that we are seeing contamination from foreground field stars. However, as mentioned, $\mathrm{HCOO}$ found the expected field star contribution to be only $\sim 5 \%$ down to the completeness limit of $K \sim 17.5$ (see $\S 1$ ). We find the apparently low-luminosity population to account for a much larger fraction $\left(\approx \frac{1}{3}\right)$ of our sample. The second possibility is that we are seeing contamination by a foreground population of $\mathrm{M}$ stars not from the field but from the surrounding OB association. The ONC (also known as Orion subgroup Id) is neighbored by three somewhat older subgroups of stars (Ia, $\mathrm{Ib}$, and Ic; see Brown et al. 1994 for a detailed description of each), which are located at distances ranging from $\sim 360$ to $400 \mathrm{pc}$ and have ages (derived from high-mass populations) from $\sim 2$ to 11.5 Myr. The most likely subgroup from which we would see contamination in our data is subgroup Ic, which is located along the same line of sight as the ONC. However, its members are thought to be only $\sim 2$ Myr old and therefore cannot account for the stars on the lower branch in the H-R diagram unless their age estimate is in error. The only known part of the OB association that could be contributing $\sim 10 \mathrm{Myr}$ old stars to our study is subgroup Ia, which is estimated to be $\sim 11.4$ Myr.

While the known high-mass population of the Ia subgroup does not extend spatially as far as the ONC, if there has been dynamical relaxation it is possible that its lower mass members may occupy a more widespread area than the OB stars. Brown et al. (1994) found the IMF for the subgroups to be a single power law of the form $\xi(\log M) \propto M^{-1.7 \pm 0.2}$ on the basis of the high-mass population $\left(M>4 M_{\odot}\right)$. Since the lower mass members have not yet been identified or studied in detail, we extrapolate the high-mass IMF to determine the total number of stars in subgroup Ia expected down to $0.02 M_{\odot}$ and find there should be $\approx 3500$ members. This number is an upper limit since we have not applied a Miller-Scalo turnover to the IMF but simply extended the power-law form. The angular size of the Orion Ia subgroup as studied by Brown et al. (1994) corresponds to a linear size of $\sim 45 \mathrm{pc}$. From this we can calculate a relaxation time for the cluster

$$
t_{\text {relax }}=n_{\text {relax }} t_{\text {cross }}=\frac{8 \ln N}{N} \frac{R}{\sigma} .
$$

Assuming a gravitationally bound cluster and a velocity dispersion of $\sigma \sim 2 \mathrm{~km} \mathrm{~s}^{-1}$, consistent with the ONC (Jones \& Walker 1988), we find a crossing time for the Orion Ia cluster of $\sim 11$ Myr and a relaxation time of $\sim 600$ Myr. Clearly, the cluster is not yet dynamically relaxed, and it is unlikely that its low-mass population would have spread significantly past its higher mass members. This calculation, however, does not rule out the possibility that the lower mass population formed over a wider spatial area than the massive stars, given that primordial mass segregation has been observed in other young clusters (e.g., Hillenbrand \& Hartmann 1998).

From the same IMF extrapolation we find the surface density of $0.5-0.02 M_{\odot}$ objects in subgroup Ia to be $\sim 2.5 \mathrm{pc}^{-2}$.
The areal extent corresponding to the angular size of our survey region (assuming a distance of $480 \mathrm{pc}$ ) is $\sim 0.5 \mathrm{pc}^{2}$. Assuming a constant surface density across this area, we would expect to find fewer than two members of Orion Ia in the current work. Even if we ignore previous age estimates, assume all three of the subgroups could be contributing to the observed lower branch of the H-R diagram, and repeat the above calculation for subgroups Ib and Ic, we would expect to see fewer than 10 stars total. Therefore, it is unlikely that the lower branch that we see in the inner ONC H-R diagram is purely due to contamination from surrounding populations. We cannot rule out that there may exist a foreground population of $\sim 10 \mathrm{Myr}$ stars that is not associated with the ONC but that was missed by the Brown et al. (1994) work because of its dearth of OB stars. However, the probability that this population would lie in exactly the same line of sight as the ONC is small.

Another possible cause of the older branch of the H-R diagram is scattered light from circumstellar disks and envelopes. Most of the objects in the lower branch of the H-R diagram have $J-H$ and $H-K$ near-infrared excesses consistent with young objects surrounded by circumstellar material that could result in their detection primarily in scattered light. If true, extinctions and, consequently, luminosities for these objects would be underestimated, making them appear older than the bulk of the population. Similar arguments have been put forth by Luhman et al. (2003b) to explain low-lying stars on the H-R diagram of IC 348. One uncertainty in this argument is that if scattered light is responsible for the apparently older population, it would have to be acting on our observations in such a way as to create a dichotomy of object luminosities rather than a continuous distribution of stars at unusually faint magnitudes. This scenario could occur, however, if the lower luminosities measured for this population are caused by a drop in observed light due to the objects being occulted by a disk. The relatively low luminosities of substellar objects would make such systems more difficult to detect, and we may be observing the brighter end of a distribution of low-mass scattered light sources. In support of this argument, one of these sources is coincident with an optically identified Hubble Space Telescope (HST) proplyd (which is known to be a photoevaporating circumstellar disk) and two with disks seen optically in silhouette only (Bally et al. 2000).

If the lower luminosity population in the inner ONC is real rather than due to systematic errors in the data reduction or analysis or contamination from the surrounding older association, the question arises of why it was not seen in previous surveys. The deepest optical photometric survey in this inner region is that by Prosser et al. (1994), which is reported complete to $V \approx 20$ and $I \approx 19 \mathrm{mag}$, although no details are given about how these numbers were derived or how they might vary with position in the nebula. One can view the photometric data of H97, which is a combination of groundbased CCD photometry out to $15^{\prime}$ from the cluster center and the Prosser et al. (1994) HST CCD photometry in the inner $3^{\prime}$ or so, in terms of CMDs binned by radial distance from the cluster center. At all radial distances there are some stars that are low in the CMD. However, it is only in radial bins beyond $\sim 7^{\prime}$ that a substantial population (although still disproportionately small compared with our current findings) of apparently 10 Myr old low-mass $\left(0.1-0.3 M_{\odot}\right)$ stars begins to appear, as might be expected if they are contaminants from the Orion Ic association. The inner radial bins do not display this 


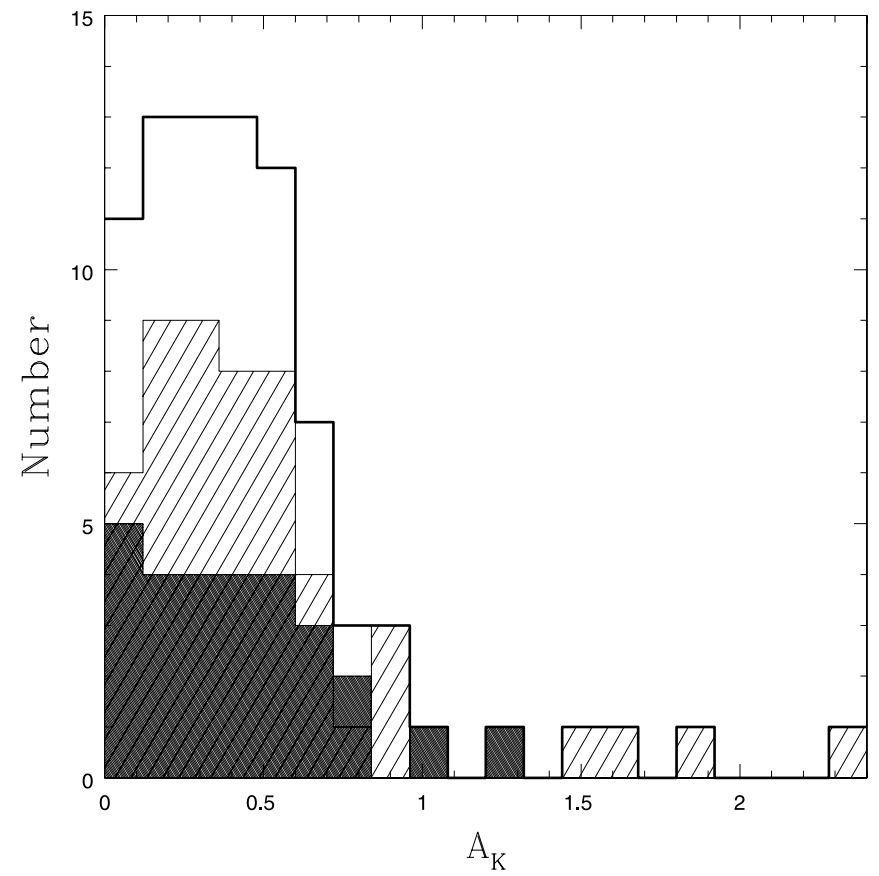

FIG. 12.-Histogram of extinction for the sources included in Fig. 11. The open histogram indicates all objects; the hatched and shaded histograms include the two populations with apparent ages (as interpreted from the H-R diagram) of $\sim 1$ and $\sim 10 \mathrm{Myr}$, respectively. We find similar extinction distributions for all three populations.

population, although it should have been detected at masses larger than $0.15 M_{\odot}$ in the absence of extinction. Notably, the apparently 10 Myr old population in Figure 11 does not lack extinction. Figure 12 shows a histogram of extinction for sources included in Figure 11. The open histogram indicates all objects; the hatched and shaded histograms include only the 1 and 10 Myr old populations, respectively. We find all three populations to have similar, roughly constant distributions for $A_{K} \lesssim 0.6\left(A_{V} \lesssim 10\right)$, giving further evidence that the lower luminosity branch of the H-R diagram is likely not a foreground population. Accounting for typical errors $(\sim 0.1$ mag at $K$ and $\sim 1.5$ mag at $V$; see $\S 4.1)$ does not change our conclusions. For extinction values larger than about 2 visual mag, the $0.15-0.5 M_{\odot}$ stars at $10 \mathrm{Myr}$ in Figure 11 would have been missed by the optical Prosser et al. (1994) photometric survey but uncovered in later infrared surveys. Further, Walker et al. (2004) find from theoretical work a lower incidence of disk occultation for higher mass CTTSs ( $\sim 20 \%$ of systems with disks) in comparison with lower mass substellar objects (up to $\sim 55 \%$ of systems with disks) because of smaller disk scale heights and less disk flaring. Therefore, if the low-luminosity objects are a population of scattered light sources, we might expect to observe smaller numbers of them at higher masses.

\section{LOW-MASS IMF}

In this section we use theoretical mass tracks and isochrones to determine a mass and age for each object in our $\mathrm{H}-\mathrm{R}$ diagram. In $\S 5.1$ we discuss our choice of models, and in $\S 5.2$, the different criteria we consider for including a star in the IMF. Finally, in $\S 5.3$ we present the derived mass function and point out important features.

\subsection{Models and Mass Estimates}

Currently, there are relatively few sets of PMS evolutionary models that extend into the substellar regime. The Lyon group (see Baraffe et al. 1998; Chabrier et al. 2000) calculations cover $0.001-1.2 M_{\odot}$ but do not extend to the large radii of stars younger than 1 Myr. Thus, they must be applied to young low-mass star-forming regions with caution. The other widely utilized set of low-mass PMS models is that by DM97. These models cover $0.017-3.0 M_{\odot}$ over an age range of $7 \times 10^{4} \mathrm{yr}$ to $100 \mathrm{Myr}$ and are therefore representative of even extremely young regions. A detailed analysis and comparison of the models is given by Hillenbrand \& White (2004). For the current work we use DM97 tracks and isochrones to determine masses and ages for stellar and substellar objects in our H-R diagram.

\subsection{Completeness}

Because the ONC is highly crowded and extremely nebulous, we must be certain that the spectroscopic sample is representative of the population as a whole. Figure 13 shows a histogram of completeness as a function of magnitude. Open and hatched histograms indicate the distribution of $\mathrm{HCOO}$

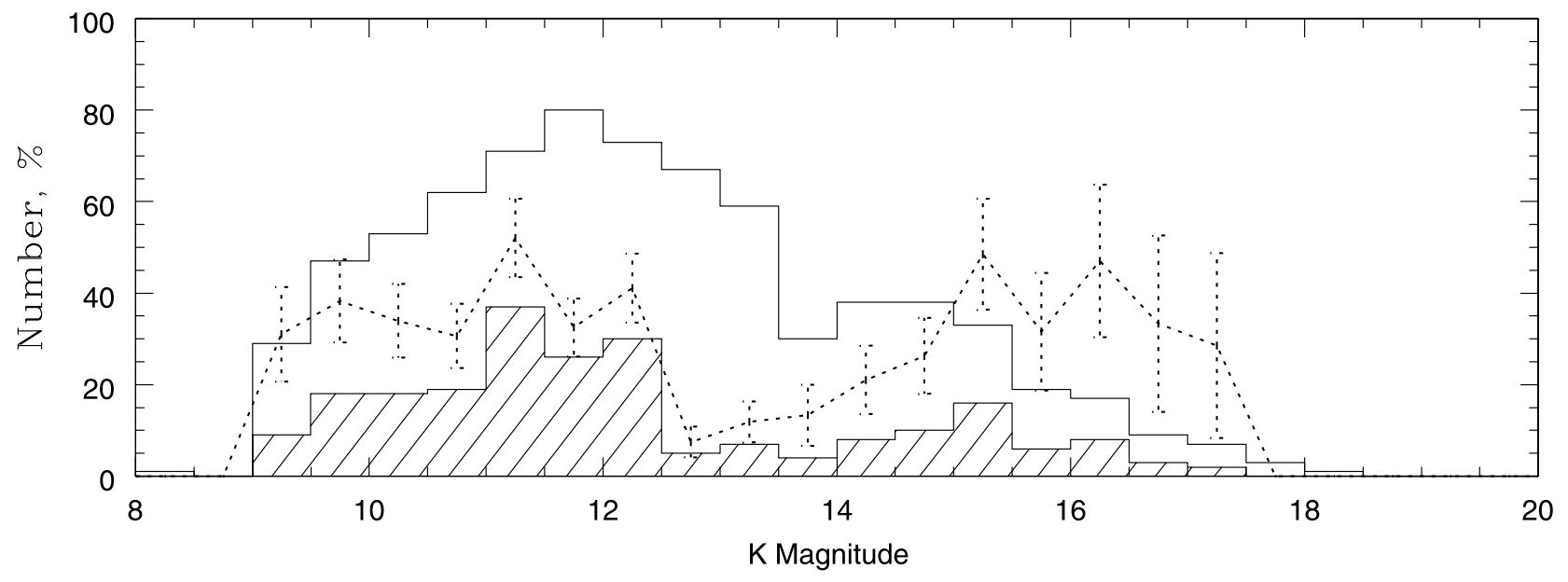

FIG. 13.- Histogram of H-R diagram completeness as a function of magnitude. Open and hatched histograms indicate the distribution of all HC00 photometry

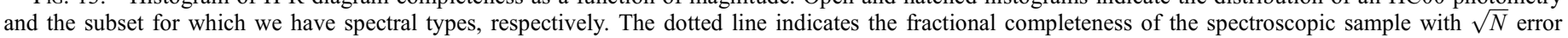

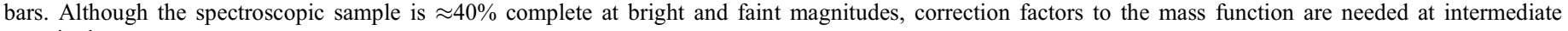
magnitudes. 


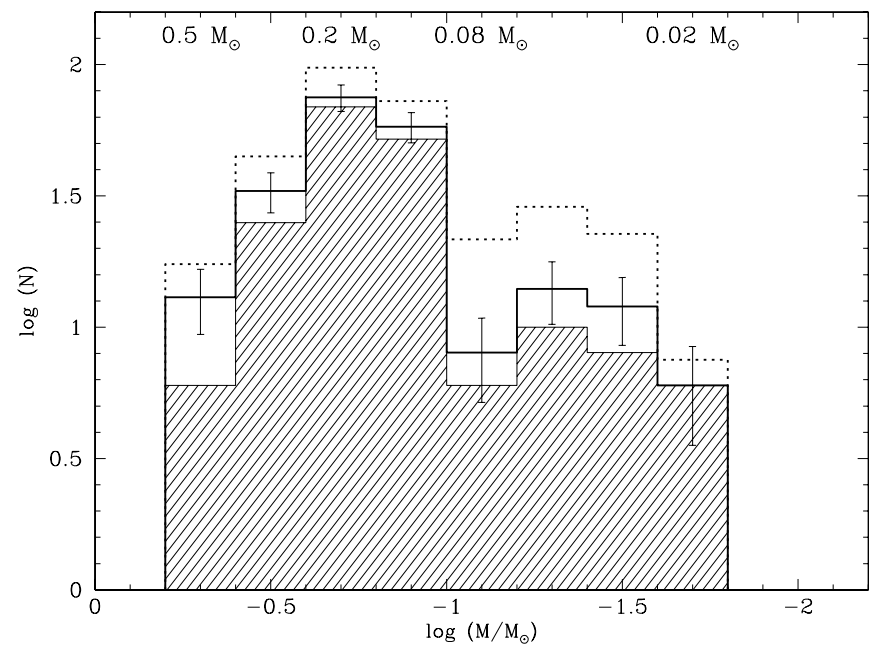

FIG. 14.-Mass function for all stars within the inner 5 ' $^{\prime} 1 \times 5$. $^{\prime} 1$ of the ONC that have spectral types later than M0 from the present work or the literature. The thick-lined open histogram shown with $\sqrt{N}$ error bars indicates all stars. The dotted open histogram represents the same sample corrected for incomplete magnitude bins (Fig. 13). The hatched histogram indicates only stars younger than $\sim 5$ Myr according to the H-R diagram (Fig. 11).

photometry and of sources for which we have spectral types, respectively. The hatched histogram includes not only sources for which we have new spectral types but also optically classified sources from the literature (H97 and references therein) that fall within our survey region and were determined to be of spectral type M0 or later. In combining the two data sets, our goal is to assemble a statistically large sample of cool, spectroscopically confirmed ONC members down to extremely low masses $\left(M \sim 0.02 M_{\odot}\right)$ from which to create our IMF.

The dotted line indicates the fractional completeness with $\sqrt{N}$ error bars. We estimate that we are $\sim 40 \%$ complete across our magnitude range with the exception of the $12.5<K<15$ bins. Undersampling at these intermediate magnitudes is caused by our combining the sample of brighter optically classified, mainly stellar objects with our sample of infraredclassified objects to observe objects fainter than the substellar limit. Therefore, magnitude bins corresponding to masses near the hydrogen-burning limit are underrepresented. We can correct for this by determining the masses that correspond to objects in the depleted magnitude bins. We then add stars to those mass bins according to the fractional completeness of the magnitude bins that they came from, relative to the overall $40 \%$ completeness. We discuss both the corrected and uncorrected IMFs in the next section.

\subsection{Stellar and Substellar IMF}

Figure 14 shows the mass function for stars within the inner $5.1 \times 5$.' 1 of the ONC that have spectral types derived from either infrared or optical spectral data later than M0. The thick-lined open histogram, shown with $\sqrt{N}$ error bars, includes all stars meeting this criteria that were not determined to have high surface gravity (see Table 1). In total, the IMF sample includes $\sim 200$ stars and contains objects with masses as high as $\sim 0.6 M_{\odot}$ and as low as less than $0.02 M_{\odot}$. Mass completeness (to the $40 \%$ level) extends from $\sim 0.4 M_{\odot}$ (corresponding to an M0 star at $1 \mathrm{Myr}$; see Fig. 11) to the completeness limit of $\mathrm{HC} 00, \sim 0.03 M_{\odot}$ for $A_{V}<10$. The dotted open histogram indicates an IMF for the same sample, in which we have corrected for the incomplete magnitude bins. The most substantial correction occurs right at the substellar limit, as indicated in $\S 5.2$. The hatched histogram represents only those objects determined from H-R diagram analysis to be younger than 5 Myr. Thus, we remove any possible bias occurring by inclusion of the apparently older $\sim 10$ Myr population that may or may not be a real part of the cluster. As can be seen, the apparently older stars constitute a relatively uniformly spaced population across the mass range we are exploring (see also Fig. 11), and including them in the IMF does not affect our interpretation.

The stellar/substellar IMF for the inner ONC in Figure 14 peaks at $\sim 0.2 M_{\odot}$ and falls across the hydrogen-burning limit into the brown dwarf regime. Using the observed IMF, we find $\sim 17 \%$ (39) of the objects in our sample to be substellar $\left(M<0.08 M_{\odot}\right)$. Below $\sim 0.08 M_{\odot}$ the mass distribution levels off through our completeness limit of $\sim 0.02 M_{\odot}$. There may be a secondary peak at $\sim 0.05 M_{\odot}$. Decreasing the bin size by $50 \%$ yields the same results, with the peak at $0.05 M_{\odot}$ becoming even more pronounced. Increasing the bin size by $50 \%$ produces a steady decline into the substellar regime. The total mass inferred between 0.6 and $0.02 M_{\odot}$ from our data is $\sim 41 M_{\odot}$, which, corrected for our $40 \%$ completeness factor, becomes $\approx 100 M_{\odot}$. This value is a lower limit because we have already shown that we are not $40 \%$ complete at all magnitudes. We find an average mass of $\sim 0.18 M_{\odot}$, corresponding to the peak in our data.

\section{DISCUSSION}

\subsection{Comparison with Previous ONC IMF Determinations}

The stellar/substellar IMF has been discussed in previous work on the ONC. A determination based on near-infrared photometric data was made by $\mathrm{HC} 00$ using $H$ and $K$ magnitudes and colors combined with star count data to constrain the IMF down to $\sim 0.03 M_{\odot}$. They find a mass function for the inner regions of the $\mathrm{ONC}$, which rises to a peak around $0.15 M_{\odot}$ and then declines across the hydrogen-burning limit, with slope $N(\log M) \propto M^{0.57}$. M02 transform the inner ONC's $K$-band luminosity function into an IMF and find a mass function that rises with decreasing mass to form a broad peak between $0.3 M_{\odot}$ and the hydrogen-burning limit before turning over and falling off into the substellar regime. This decline is broken between 0.02 and $0.03 M_{\odot}$, at which the IMF may contain a secondary peak near the deuterium-burning limit of $\sim 13 M_{\odot}$. Luhman et. al (2000) combined near-infrared NICMOS photometry of the inner $140^{\prime \prime} \times 140^{\prime \prime}$ of the ONC with limited ground-based spectroscopy of the brightest objects $(K<12)$ to determine a mass function that follows a power-law slope similar to but slightly steeper than the Salpeter value, until it turns over at $\sim 0.2 M_{\odot}$ and declines steadily through the brown dwarf regime. H97 presents the most extensive spectroscopic survey of the ONC, combining optical spectral data with $V$ - and $I$-band photometry over a large area $\left(\sim 2.5 \mathrm{pc}^{2}\right)$ extending into the outer regions of the cluster. The IMF determination covers a large spectral range and appears to be rising from the high-mass $\left(50 M_{\odot}\right)$ to lowmass $\left(0.1 M_{\odot}\right)$ limits of that survey.

The peak in our IMF, $\sim 0.2 M_{\odot}$, matches remarkably well those found from both the deep near-infrared imaging IMF studies ( $\mathrm{HCOO}$ and M02), which cover similar survey areas, and the Luhman et al. (2000) study, which covered only the very inner region of the cluster. Our data also show a leveling off in the mass distribution through the substellar regime 
similar to that found by HC00. A significant secondary peak within the substellar regime has been claimed by M02. While we see some evidence for such a peak in our data, this result is not robust to within the errors. Furthermore, if it is real, we find that the secondary peak occurs at a slightly higher mass than that in $\mathrm{M} 02\left(\sim 0.05 M_{\odot}\right.$ vs. $\left.\sim 0.025 M_{\odot}\right)$. The primary difference between the observed IMF derived in the current work and those presented in previous studies of the substellar ONC population is the steepness of the primary peak and the sharpness of the falloff at the hydrogen-burning limit (see for comparison Fig. 16 of M02). Most IMF determinations for this region exhibit a gradual turnover in the mass function around $\sim 0.2 M_{\odot}$ until $\approx \frac{2}{3}$ the level of the primary peak is reached, at which point the IMF levels off or forms a secondary peak. However, we find a sharp falloff beyond $\sim 0.1 M_{\odot}$ to $\approx \frac{1}{2}$ the primary peak value.

\subsection{Photometric versus Spectroscopic Mass Functions}

As mentioned in $\S 1$, spectroscopy is needed to study a cluster's IMF in more than a statistical sense. The fact that many of the fainter objects in our survey that were expected to be substellar on the basis of their location in the $K,(H-K)$ diagram are in fact hotter, possibly older objects is strong evidence in support of this statement. Independent knowledge of an individual star's age, extinction, and infrared excess arising from the possible presence of a circumstellar disk is needed before definitive conclusions can be drawn about that object's mass. Despite the increasing number of brown dwarfs studied, both in the field and in clusters, details of their formation process remain sufficiently poorly understood that knowledge of near-infrared magnitudes and colors alone is not enough to accurately predict these characteristics for young low-mass objects. Neither can near-infrared magnitudes alone distinguish between cluster members and nonmembers, and field star contamination must be modeled rather than accounted for directly.

Previous studies of the ONC had spectroscopy available in general only for the stellar population and relied on photometry alone to determine the mass function at substellar masses. This may have caused overestimates in the number of brown dwarfs for the reasons discussed above. However, the more significant cause of the shallower peak in previous IMF determinations for the inner ONC as opposed to the sharply peaked IMF derived here is likely just the inherent nature of photometric versus spectroscopic mass functions. Determining masses for stars in a sample from temperatures derived from spectral types necessarily discretizes the data. Conversely, photometric studies are by their nature continuous distributions, and deriving masses from magnitudes and colors or luminosity functions results in a smooth distribution of masses. We emphasize that while neither situation is ideal, mass functions derived for young objects from infrared photometry alone represent only the most statistically probable distribution of underlying masses. Spectroscopy is needed to derive cluster membership and masses for individual objects.

\subsection{Comparison with Other Star-forming Regions}

Diagnostic studies of stellar populations in different locations and at varying stages of evolution are needed to explore the possibility of a universal mass function. While one might expect that the IMF should vary with the star formation environment, we do not yet have enough evidence to determine whether such a variation exists. Aside from work already mentioned on the ONC, numerous studies have been carried out to characterize the low-mass stellar and substellar mass functions of other young clusters in various environments. Because of the intrinsic faintness of these objects, most surveys are photometric. Authors then use a combination of theoretical models and statistical analysis to transform a cluster's CMD or luminosity function into an IMF that may or may not accurately represent the underlying cluster population (see $\S 6.2)$.

However, the substellar populations of two other young star-forming clusters have been studied spectroscopically using techniques similar to those presented here. Luhman (2000), Briceño et al. (2002), and Luhman et al. (2003a) surveyed the sparsely populated Taurus star-forming region, and Luhman et al. (2003b) studied the rich cluster IC 348. These clusters have ages similar to the ONC (1 and 2 Myr). Therefore, if the IMF is universal, similar mass distributions should be observed for all three clusters. Contrary to this hypothesis, Luhman et al. (2003b) discuss the very different shapes of the substellar mass distributions in Taurus and IC 348. The IMF for Taurus peaks around $\sim 0.8 M_{\odot}$ and then declines steadily to lower masses through the brown dwarf regime. The IC 348 mass function rises to a peak around $0.15 M_{\odot}$ and then falls off sharply and levels out for substellar objects. While direct comparison of the data requires caution given that different mass tracks were used for the two studies (Luhman et al. 2003b used Baraffe et al. 1998 tracks, whereas we used DM97 tracks to infer masses), we find our that IMF for the ONC bears a remarkable resemblance to that presented for IC 348 in Luhman et al. (2003b; see for comparison Fig. 12 in Luhman et al. 2003b). Both IMFs peak at $\sim 0.15-0.2 M_{\odot}$ and fall off rather abruptly at the substellar boundary. The fact that the IMFs derived for these two dense young clusters bear such a close resemblance to each other while exhibiting distinguishable differences from the IMF determined for the much more sparsely populated Tau cluster gives strong support to the argument put forth by authors such as Luhman et al. (2003b) that the IMF is not universal but may instead depend on the star formation environment. Alternatively, Kroupa et al. (2003) argue through numerical simulation that observed differences in the Taurus and ONC stellar mass functions could be due to dynamical effects operating on initially identical IMFs; however, their model does not reproduce the observed differences in substellar mass functions without invoking different initial conditions (e.g., turbulence; Delgado-Donate et al. 2004).

Previous studies such as those mentioned above have found much higher brown dwarf fractions for the ONC in comparison with other clusters such as Taurus and IC 348 (see Luhman et al. 2003b). Briceño et al. (2002) define the ratio of the numbers of substellar and stellar objects as

$$
R_{S S}=\frac{N\left(0.02 M_{\odot} \leq M \leq 0.08 M_{\odot}\right)}{N\left(0.08 M_{\odot}<M \leq 10 M_{\odot}\right)} .
$$

We have recomputed these numbers for Taurus and IC 348 using the DM97 models and find values of $R_{S S}=0.11$ (Taurus) and $R_{S S}=0.13$ (IC 348). These values are very close to those found by Luhman et al. (2003b) using the Baraffe et al. (1998) models: $R_{S S}=0.14$ (Taurus) and $R_{S S}=0.12$ (IC 348). In contrast, Luhman et al. (2000) find a value of $R_{S S}=0.26$ from primarily photometric work on the inner ONC (using Baraffe et al. 1998 models). Considering the spectroscopic IMF presented here (Fig. 14), we find a lower value of $R_{S S}=0.20$, indicating that although the ONC may have a higher brown 
dwarf fraction than Taurus or IC348, it is lower than previously inferred from photometric studies.

\section{SUMMARY}

We have identified for the first time a large sample of spectroscopically confirmed young brown dwarfs within the inner regions of the ONC, including five newly classified M9 objects. From these data we have made an H-R diagram and possibly discovered a previously unknown population of $\sim 10$ Myr old low-mass stars in the inner regions of the cluster. We have examined this population in detail and determined that it is not an artifact arising from systematic errors in the spectral reduction or classification processes. We have ruled out that this population consists primarily of "contaminants" from the surrounding OB1 association. If this population is real, we propose that it was not detected in previous work because of its intrinsic faintness, coupled with extinction effects. Another possible scenario is that these objects constitute a population of scattered light sources. If this hypothesis is correct, these objects provide indirect observational support for recent theoretical arguments indicating higher disk occultation fractions for young substellar objects in comparison with higher mass CTTSs (Walker et al. 2004).

From the H-R diagram we have used DM97 tracks and isochrones to infer a mass for each object and constructed the first spectroscopic substellar $\left(0.6 M_{\odot}>M>0.02 M_{\odot}\right)$ IMF for the ONC. Our mass function peaks at $\sim 0.2 M_{\odot}$, consistent with previous IMF determinations (M02; HC00; Luhman et al. 2000); however, it drops off more sharply past $\sim 0.1 M_{\odot}$, before roughly leveling off through the substellar regime. We compare our mass function with those derived for Taurus and IC 348, which also use spectroscopic data, and find remarkable agreement between mass distributions found in IC 348 and the ONC.

The authors would like to thank Davy Kirkpatrick for useful comments, which improved the final paper, and Kevin Luhman and Lee Hartmann for their insights and suggestions, which helped in our analysis. We thank Alice Shapley and Dawn Erb for sharing their method of subtracting sky lines from NIRSPEC images, as well as NIRSPEC support astronomers Greg Wirth, Grant Hill, and Paola Amico for their guidance during observing. We are also appreciative of Michael Meyer for his participation in the CRSP data acquisition and of assistance at the KPNO $4 \mathrm{~m}$ telescope from Dick Joyce. Finally, we thank LRIS observers N. Reid and B. Schaefer for obtaining several spectra for us. C. L. S. acknowledges support from a National Science Foundation Graduate Research Fellowship.
Bally, J., O’Dell, C. R., \& McCaughrean, M. J. 2000, AJ, 119, 2919

Baraffe, I., Chabrier, G., Allard, F., \& Hauschildt, P. H. 1998, A\&A, 337, 403

Bessell, M. S. 1991, AJ, 101, 662

Bessell, M. S., \& Brett, J. M. 1988, PASP, 100, 1134

Briceño, C., Luhman, K. L., Hartmann, L., Stauffer, J. R., \& Kirkpatrick, J. D. 2002, ApJ, 580, 317

Brown, A. G. A., de Geus, E. J., \& de Zeeuw, P. T. 1994, A\&A, 289, 101

Cardelli, J. A., Clayton, G. C., \& Mathis, J. S. 1989, ApJ, 345, 245

Carpenter, J. M., Hillenbrand, L. A., \& Strutskie, M. F. 2001, AJ, 121, 3160

Chabrier, G., Baraffe, I., Allard, F., \& Hauschildt, P. 2000, ApJ, 542, 464

Cohen, J. G., Frogel, J. A., Persson, S. E., \& Elias, J. H. 1981, ApJ, 249, 481

D’Antona, F., \& Mazzitelli, I. 1997, Mem. Soc. Astron. Italiana, 68, 807 (DM97)

Dahn, C. C., et al. 2002, AJ, 124, 1170

Delgado-Donate, E. J., Clarke, C. J., \& Bate, M. R. 2004, MNRAS, 347, 759 Gorlova, N. I., Meyer, M. R., Rieke, G. H., \& Liebert, J. 2003, ApJ, 593, 1074

Hillenbrand, L. A. 1997, AJ, 113, 1733 (H97)

Hillenbrand, L. A., \& Carpenter, J. M. 2000, ApJ, 540, 236 (HC00)

Hillenbrand, L. A., \& Hartmann, L. W. 1998, ApJ, 492, 540

Hillenbrand, L. A., Strom, S. E., Calvet, N., Merrill, K. M., Gatley, I., Makidon, R. M., Meyer, M. R., \& Skrutskie, M. F. 1998, AJ, 116, 1816

Hillenbrand, L. A., \& White, R. J. 2004, ApJ, 604, 741

Jones, B. F., \& Walker, M. F. 1988, AJ, 95, 1755

Joy, A. H. 1949, ApJ, 110, 424

Kleinmann, S. G., \& Hall, D. N. B. 1986, ApJS, 62, 501

Kroupa, P., Bouvier, J., Duchêne, G., \& Moraux, E. 2003, MNRAS, 346, 354

Lada, C. J., Muench, A. A., Haisch, K. J., Lada, E. A., Alves, J., Tollestrup, E. V., \& Willner, S. P. 2000, AJ, 120, 3162

Leggett, S. K., Allard, F., Berriman, G., Dahn, C. C., \& Hauschildt, P. H. 1996, ApJS, 104, 117

Leggett, S. K., et al. 2002, ApJ, 564, 452

\section{EFERENCES}

Luhman, K. L. 2000, ApJ, 544, 1044

Luhman, K. L., Briceño, C., Stauffer, J. R., Hartmann, L., Barrado y Navascués, D., \& Nelson, C. 2003a, ApJ, 590, 348

Luhman, K. L., Reike, G. H., Young, E. T., Cotera, A. S., Chen, H., Rieke, M. J., Schneider, G., \& Thompson, R. I. 2000, ApJ, 540, 1016

Luhman, K. L., Stauffer, J. R., Muench, A. A., Rieke, G. H., Lada, E. A., Bouvier, J., \& Lada, C. J. 2003b, ApJ, 593, 1093

McGovern, M. R., Kirkpatrick, J. D., McLean, I. S., Burgasser, A. J., Prato, L., \& Lowrance J. 2004, ApJ, 600, 1020

McLean, I. S., McGovern, M. R., Burgasser, A. J., Kirkpatrick, J. D., Prato, L., \& Kim, S. S. 2003, ApJ, 596, 561

McLean, I. S., et al. 2000, ApJ, 533, L45

Meyer, M. R., Calvet, N., \& Hillenbrand, L. A. 1997, AJ, 114, 288

Muench, A. A., Alves, J., Lada, C. J., \& Lada, E. A. 2001, ApJ, 558, L51

Muench, A. A., Lada, E. A., Lada, C. J., \& Alves, J. 2002, ApJ, 573, 366 (M02)

Prosser, C. F., Stauffer, J. R., Hartmann, L. W., Soderblom, D. R., Jones, B. F., Werner, M. W., \& McCaughrean, M. J. 1994, ApJ, 421, 517

Reid, I. N., Burgasser, A. J., Cruz, K. L., Kirkpatrick, J. D., \& Gizis, J. E. 2001, AJ, 121,1710

Reid, I. N., et al. 1999, ApJ, 521, 613

Salpeter, E. E. 1955, ApJ, 121, 161

Tokunaga, A. T., \& Kobayashi, N. 1999, AJ, 117, 1010

Wainscoat, R. J., Cohen, M., Volk, K., Walker, H. J., \& Schwartz, D. E. 1992, ApJS, 83,111

Walker, C., Wood, K., Lada, C. J., Robitaille, T., Bjorkman, J. E., \& Whitney, B. 2004, MNRAS, in press (astro-ph 0403276)

Wilking, B. A., Greene, T. P., \& Meyer, M. R. 1999, AJ, 117, 469

Wilking, B. A., Meyer, M. R., Greene, T. P., Mikhail, A., \& Carlson, G. 2004, AJ, 127, 1131 


\section{ERRATUM: “THE SPECTROSCOPICALLY DETERMINED SUBSTELLAR MASS FUNCTION OF THE ORION NEBULA CLUSTER" (ApJ, 610, 1045 [2004])}

Catherine L. Slesnick, Lynne A. Hillenbrand, and John M. Carpenter

Department of Astronomy, California Institute of Technology

In Table 1 object HC 722 was incorrectly classified as an L0 dwarf. The original classification was based on the apparent prominence in the spectrum of the K I doublet ( 7700 ̊). However, standard stars available to us at the time were of lower resolution (by a factor of 2) than the program stars. Narrow spectral features such as the K I doublet were thus blurred and appeared weaker. We have since reanalyzed the spectrum of object HC 722 in comparison to higher resolution standards taken with LRIS (the same instrument used to obtain the spectrum of HC 722), and reclassified it as an M6.5 V dwarf object. In Table 4 the values for HC 722 should be replaced by $\log T_{\text {eff }}=3.429 \mathrm{~K}, \log \left(L / L_{\odot}\right)=-2.89$, and $M=0.04 M_{\odot}$.

Note that the object is still considered a dwarf, showing no signs of lower gravity. This, combined with the fact that we derive zero $A_{V}$ for $\mathrm{HC} 722$, implies that it is likely a foreground field dwarf. If we assume an absolute magnitude $M_{K}=9.85 \mathrm{mag}$ (S. K. Leggett, ApJS, 82, 351 [1992]) for an M6.5 V star, we derive a distance modulus of 7.58 ( $d \sim 330 \mathrm{pc}$ ). Because HC 722 was previously determined to have higher gravity than members of the ONC, it was not included in the derivation or analysis of the cluster IMF. As this "high gravity" assignment is not affected by the reclassification, the change does not alter or impact any of our original conclusions. 